\title{
Familienpolitik, Bildung und Berufskarrieren von Müttern in Deutschland, USA und Schweden
}

\author{
Daniela Grunow $•$ Silke Aisenbrey $•$ Marie Evertsson
}

Zusammenfassung: Dieser Beitrag widmet sich der beruflichen Entwicklung von Müttern mit ungleichen Bildungsressourcen in international vergleichender Perspektive. Wir untersuchen die Strategien zur Vereinbarkeit von Berufskarrieren und Elternschaft in Deutschland, USA und Schweden für Frauen mit unterschiedlichem Schulbildungsniveau. Die Analysen beruhen auf länderspezifischen Längsschnittdaten (GLHS-West, NLSY, LNU). Mit Hilfe ereignisanalytischer Verfahren untersuchen wir, wie lange Mütter mit unterschiedlichem Bildungsniveau nach der Geburt eines Kindes ihre Erwerbstätigkeit unterbrechen, ob die Rückkehr, so, wie in Elternzeit-Programmen vorgesehen, auf dem vorherigen beruflichen Prestigeniveau erfolgt und unter welchen Umständen Müttern mit hohem, mittleren und niedrigen Schulbildungsniveau der berufliche Statuserhalt gelingt. Die hoch gebildeten Mütter zeigen in allen drei Ländern die stärkste Arbeitsmarktbindung. In den USA sind fast die Hälfte der Mütter mit College Ausbildung kontinuierlich erwerbstätig, gegenüber einem Viertel der Mütter ohne High School Diplom. In Schweden unterbrechen Mütter für mindestens ein halbes Jahr, unabhängig vom Bildungsniveau. In Deutschland verzichtet ein Viertel der Mütter mit (Fach-)Abitur auf Elternzeit. Konsequenzen einer gesetzlichen Ausweitung der Unterbrechungszeiten sind in Deutschland und Schweden weitgehend bildungsunabhängig. In den USA kehren mittel- und hochqualifizierte Mütter nach Einführung des FMLA langsamer auf gleichwertige Positionen zurück.

Schlüsselwörter: Berufsmobilität $\cdot$ Mütter $\cdot$ Erwerbskarriere $\cdot$ Lebenslauf $\cdot$ Internationaler Vergleich · Ereignisanalyse

(C) Die Autor(en) 2011. Dieser Artikel ist auf Springerlink.com mit Open Access verfügbar.

D. Grunow $(\square)$

Universiteit van Amsterdam,

Oudezijds Achterburgwal 185, 1012 Amsterdam, Niederlande

E-Mail: d.grunow@uva.nl

S. Aisenbrey

Yeshiva University, 500 West 185th Street, 547 Belfer Hall,

New York, NY 10033, USA

M. Evertsson

Swedish Institute for Social Research Stockholm University,

SE-10691 Stockholm, Schweden 


\title{
Motherhood, family policy, education, and careers in Germany, the U.S., and Sweden
}

\begin{abstract}
This article compares the duration of and consequences for employment interruptions for mothers with distinct educational qualifications in cross-national perspective. We analyze the duration of mothers' employment interruption and the relative quality of the job after childbirth, for high and low qualified mothers in Germany, Sweden, and the United States. Analyses are based on harmonized national longitudinal data sets (GLHS-West, NLSY, LNU), which allow us to study mothers' careers over the past 30-35 years. We apply event-history analysis to study how long mothers with distinct educational levels interrupt employment after childbirth, and how the patterns of return to work differ with respect to mothers' educational level. In all three countries the highly educated display a greater labor market attachment. In the US, almost half of the mothers with some college background, compared to only a quarter of high-school dropouts stay employed continuously after childbirth. In Sweden, basically all mothers take time-out for at least half a year. In Germany, 25\% of mothers with university entrance qualification do not claim parental leave. In Germany and Sweden consequences of leave extensions seem to operate independent of mothers' educational levels. In the U.S. medium and highly educated mothers tend to return more slowly after the enactment of the FMLA.
\end{abstract}

Keywords: Occupational mobility $\cdot$ Mothers $\cdot$ Employment career $\cdot$ Life course International comparison · Event history analysis

\section{Einleitung}

Mutterschutz- und Elternzeiten gelten als wichtiges familienpolitisches Instrument zur Unterstützung der Erwerbstätigkeit von Frauen. Im internationalen Vergleich zeigen sich große Unterschiede hinsichtlich der Länge und der finanziellen Absicherung von Elternzeiten sowie im Zugang zu diesen Rechten und Leistungen. Während viele Staaten, darunter auch Westdeutschland, jahrzehntelang speziell auf die Ausdehnung dieser gesetzlich geschützten Unterbrechungen setzten, werden in jüngster Zeit zunehmend Zweifel an der arbeitsmarktbindenden und berufssichernden Wirkung gesetzlicher Sorgezeiten laut. Speziell in Ländern, die Anreize zu langen Erwerbsunterbrechungen geben, unterbricht ein großer Teil der Mütter deutlich länger als gesetzlich erwünscht und unterstützt (Aisenbrey et al. 2009). Unklar ist jedoch, wie sich spezifische Regelungen auf die berufliche Karriere von Müttern mit unterschiedlichem Bildungsniveau auswirken und ob ein Zusammenhang mit der Inanspruchnahme oder der Dauer von Elternzeit in Abhängigkeit vom Bildungsniveau der Mütter besteht. Hier besteht Klärungsbedarf; nicht zuletzt weil der Anteil an hoch qualifizierten Frauen in den Industrieländern in den letzten Jahrzehnten enorm angestiegen ist. Führt die Ausweitung von Elternzeitregelungen in der Praxis zu mehr beruflicher Instabilität und Abwärtsmobilität in bestimmten Bildungsschichten oder ermöglichen Jobgarantien Müttern berufliche Kontinuität für alle, auch nach längeren Auszeiten? Greifen Elternzeitregelungen unabhängig vom Bildungsniveau oder verstärken die erweiterten Optionen der Erwerbsunterbrechung die ohnehin bestehenden sozialen Unterschiede zwischen den gut ausgebildeten und den gering qualifizierten Müttern?

Um die Wirkungsweise verschiedener Elternzeitregelungen auf die weitere berufliche Entwicklung von Müttern mit unterschiedlichen Bildungsniveaus besser einschätzen zu können, fehlt es derzeit an international vergleichenden Längsschnittstudien, die einen 
Vergleich von Elternzeitregelungen, Erwerbsunterbrechungsmustern und deren Konsequenzen über die Zeit erlauben. ${ }^{1}$ Diese Forschungslücke stellt den Ausgangspunkt unserer Untersuchungen dar. Die hier präsentierten Ergebnisse sind Teil einer umfassenden Studie zu den Konsequenzen von Erwerbsunterbrechungen für Frauen (Aisenbrey et al. 2009). In dem vorliegenden Beitrag konzentrieren wir uns auf die bildungsspezifischen Konsequenzen von Erwerbsunterbrechungen für Mütter. Wir untersuchen erstens, wie sich die Ausdehnung von Elternzeitregelungen in den 1980er und 1990er Jahren in Westdeutschland, Schweden und den USA auf die Unterbrechungsdauer von Müttern mit unterschiedlichem beruflichen Status und Bildungsniveau niedergeschlagen hat und zweitens, ob negative Konsequenzen, die sich aus einer Erwerbsunterbrechung für Mütter ergeben, selektiv oder universell von Elternzeitregelungen abgefedert werden. Empirisch untersuchen wir zunächst, wie lange erwerbstätige Mütter in den drei Ländern in Abhängigkeit der jeweils geltenden Mutterschutz- oder Elternzeitregelungen nach der Geburt eines Kindes ihre Beschäftigung unterbrechen und wie sich diese Unterbrechungsdauern nach dem Bildungsniveau unterscheiden. Danach analysieren wir, ob für bestimmte Bildungsgruppen die Rückkehr in den Arbeitsmarkt mit erhöhtem Risiko eines Statuswechsels einhergeht. Schließlich untersuchen wir die Interaktion der gesetzlichen Verlängerung der Elternzeit mit dem Bildungsniveau von Müttern. Unser besonderes Augenmerk in allen Analysen gilt den Bildungseffekten, da gesetzliche Jobgarantien für Frauen in Elternzeit prinzipiell unabhängig vom Bildungsniveau greifen sollten, Erwerbsunterbrechungen jedoch wahrscheinlich nach Bildung und Berufsposition stratifiziert sind.

Da sich der größte Teil der Studien aus diesem Bereich primär mit Lohneinbußen beschäftigt und Bildung vor allem als Kontrollvariable einbezieht (Albrecht et al. 1999; Baum 2002; Budig und England 2001; Datta Gupta und Smith 2002; Gangl und Ziefle 2009; Lundberg und Rose 2000; Ziefle 2004), wissen wir wenig darüber, ob sich kinderbedingte Erwerbsunterbrechungen auf den beruflichen Status von Müttern verschiedener Bildungsschichten unterschiedlich auswirken. Eine Analyse des Erwerbseinkommens muss zudem, speziell in Ländern mit starker Orientierung am Ernährermodell, das Haushaltseinkommen berücksichtigen und lässt so nur sehr begrenzt Rückschlüsse auf die zukünftigen Chancen von Müttern zu, ihren Unterhalt selbst zu bestreiten. Dieser Aspekt der Kommodifizierung ist unmittelbar sozialpolitisch relevant; beispielsweise angesichts der aktuellen Änderungen im deutschen Scheidungsrecht, das von Frauen nach einer Ehescheidung sehr viel schneller und in deutlich größerem Umfang als bisher die berufliche Eigenständigkeit verlangt. Um diese Chancen auf berufliche Eigenständigkeit zu beschreiben, untersuchen wir berufliche Stabilität oder Mobilität, gemessen an Veränderungen des Berufsprestiges (Treiman 1977). Die Betrachtung des Berufsprestiges als abhängige Variable misst dabei das langfristig arbeitsmarktrelevante Kapital von Frauen. Im Unterschied zum Einkommen oder Verdienst, die im Erwerbsleben situationsbeding-

1 Im Folgenden fassen wir die unterschiedlichen nationalen Regelungen und Terminologien mit dem Begriff Elternzeit zusammen. In Abschn. 2 diskutieren wir dann im Einzelnen die länderspezifischen Unterschiede beim Zugang zu und bei der Vergütung von den unterschiedlichen Mutterschutz- und Elternzeitregelungen sowie deren Veränderungen über den untersuchten Zeitraum. Der Umstand, dass unterschiedliche Regelungen in unterschiedlichem Maße auch Lohnersatzfunktionen erfüllen, motiviert länder- und periodenspezifische Unterbrechungsmuster bei Müttern. Da wir an den faktischen Erwerbsunterbrechungsmustern interessiert sind, beobachten wir Unterbrechungszeiten auch über die gesetzlich geregelten Fristen hinausgehend. 
ten Schwankungen unterworfen sein können, misst das Berufsprestige, inwiefern Frauen nach der Geburt eines Kindes der berufliche Statuserhalt gelingt. Der berufliche Statuserhalt ist eine Voraussetzung dafür, dass Mütter ihre bis dahin getätigten berufsspezifischen Bildungsinvestitionen und Arbeitsmarkterfahrungen später im Lebenslauf verwerten können.

Unsere Daten decken den historischen Zeitraum von den 1970er Jahren bis kurz nach der Jahrtausendwende ab. Die ländervergleichenden Analysen richten sich besonders auf die Politikveränderungen der 1980er und 1990er Jahre, in denen gesetzliche Regelungen zur Erwerbsunterbrechung von Müttern deutlich ausgebaut wurden. In allen drei Ländern ist das Schulbildungsniveau von Frauen und Müttern in dieser Zeit angestiegen. Die zentrale Frage in diesem Beitrag ist, ob sich angesichts der familienpolitischen Unterschiede zwischen den Ländern und über die Zeit die Differenzen in den Erwerbsbiografien von hoch und gering qualifizierten Müttern eher verringert oder weiter stratifiziert haben.

\section{Deutschland, Schweden und USA im Vergleich}

In den letzten Jahrzehnten hat sich das alte Ernährer- und Hausfrauenmodell in allen Industrieländern zugunsten einer stärkeren Erwerbsbeteiligung von Frauen gewandelt (Leitner et al. 2004; Lewis 2001). Unterschiedliche wohlfahrtsstaatliche Orientierungen hinsichtlich der Eingliederung von Frauen in den Arbeitsmarkt als soziale Sicherungsmaßnahme (Kommodifizierung) und ihrer gleichzeitigen (De-)familialisierung haben jedoch zu höchst unterschiedlichen länderspezifischen Politiken und Mustern der Erwerbsbeteiligung, speziell bei Müttern geführt (Mandel und Shalev 2009a). Deutschland gilt hier als ein klassischer Vertreter des männlichen Ernährer- oder Hausfrauenmodells, in dem unbezahlte Sorgearbeit von Ehefrauen indirekt staatlich gefördert und subventioniert wird (Ostner und Lewis 1998). Der Subventionseffekt zeigt sich empirisch auch für hoch qualifizierte Frauen, für die es in Deutschland nach wie vor schwierig ist, eine Vollzeitbeschäftigung aufzunehmen (Aisenbrey 2009). Das deutsche Politikmodell ist durch verschiedene Reformen seit den 1990er Jahren, wie das Recht des Kindes auf einen (halbtags-)Kindergartenplatz seit 1993, die Einführung flexibler und teilzeitfreundlicherer Elternzeit seit 2001 sowie die Einführung von Steuerfreibeträgen für Haushaltshilfen und Kinderbetreuung seit 2002, zunehmend uneindeutiger geworden (Leitner et al. 2004). Trotz dieser Neuerungen, die Müttern kleiner Kinder die Erwerbstätigkeit erleichtern, blieben Elemente, die das Ernährermodell fördern, im deutschen System erhalten. Dazu gehören vor allem die Kombination aus Ehegattensplitting, Teilzeitschulen und -kindergärten sowie das geschlechtsspezifisch segregierte Berufsbildungssystem, welches berufliche Segregationsprozesse in frühen Lebensphasen begünstigt und im weiteren Lebenslauf untermauert (Krüger 2003). In diesem Kontext, so wird angenommen, werden die Lebensentwürfe von Frauen durch geltende politische Rahmenbedingungen selektiv eingeschränkt und erweitert (Leitner et al. 2004), mit unklaren Folgen für hochund gering qualifizierte Frauen und Mütter. Einerseits sind es in Deutschland gerade die höheren sozialen Schichten, die sich das Hausfrauenmodell leisten können und von den Steuererleichterungen des Ehegattensplittings besonders profitieren. Andererseits bieten 
die neuen gesetzlichen Regelungen gerade für hochqualifizierte Mütter Anreize und Möglichkeiten zur beruflichen Kontinuität.

Schweden wird als sozialdemokratischer Wohlfahrtsstaat aufgrund seiner generösen Elternzeitregelungen und seiner qualitativ hochwertigen und flächendeckenden staatlichen Kinderbetreuungssysteme häufig als Kontext mit vergleichsweise guter Vereinbarkeit von Erwerbstätigkeit und Mutterschaft gesehen (Misra et al. 2007). Der Zugang zu Elternzeitregelungen setzt jedoch eine längerfristige vorhergehende Erwerbstätigkeit voraus und die weitgehend einkommensabhängige Vergütung von Elternzeiten wirkt zusätzlich als Anreiz für Mütter, sich vor der Familiengründung auf dem Arbeitsmarkt zu etablieren. Gleichzeitig ist die Rolle des Staates bei der Bereitstellung von Dienstleistungen, die anderswo primär von zumeist unbezahlten weiblichen Familienmitgliedern oder vom Markt verrichtet werden, hoch (Mandel und Shalev 2009a). Entsprechend gilt die Erwerbsbeteiligung von Frauen und Müttern in Schweden als universell und damit als unabhängig vom Bildungsniveau.

Im Gegensatz zu den vielfältigen Regelungen im deutschen und schwedischen System gilt die Rolle des us-amerikanischen Staates bei der (De-)Kommodifizierung und (De-)Familialisierung als äußerst zurückhaltend (Mandel und Shalev 2009a). In den USA ist die soziale Sicherung primär durch den Markt und über Erwerbsarbeit organisiert (Esping-Andersen 1990). Illustrativ hierfür ist zum Beispiel, dass der individuelle Zugang zu Pensionen und zur Krankenversicherung in hohem Maße über sogenannte „benefits“ funktioniert, die an ein Beschäftigungsverhältnis, vor allem in großen Firmen, geknüpft sind. Gleichzeitig sind viele US-Amerikaner von diesen Leistungen, speziell in bestimmten Lebensphasen, ausgeschlossen, da nicht alle Jobs diese „benefits“ bieten. Auch bezahlte Elternzeiten, die in Deutschland und Schweden Teil der staatlichen Politikpakete sind, sind in den USA häufig Teil dieser an den Job gebundenen Leistungen. Das Fehlen staatlicher Unterstützungsleistungen ist lange Zeit stark kritisiert worden (Gornick und Meyers 2003). Neuerdings mehren sich jedoch Stimmen, die eben diese Zurückhaltung in der intervenierenden Familienpolitik als förderlich für die Geschlechtergleichheit im Haushalt und auf dem Arbeitsmarkt sehen; speziell in den höheren Berufs- und Einkommensschichten (Mandel und Shalev 2009a). Die Zurückhaltung in Sachen Familienpolitik wird nicht als fehlende Unterstützung von Familien interpretiert, sondern als Sozialpolitik eines Gender Regimes mit dem Ziel der Geschlechtergleichheit. Hervorgehoben wird, dass die USA im internationalen Vergleich Vorreiter sind im Hinblick auf Antidiskriminierungsmaßnahmen und führend im Hinblick auf die Integration von Frauen in traditionell männliche Berufsfelder, das Management und Universitäten (Orloff 2009). In diesem Kontext gilt es auch zu beachten, dass die vorhandenen Elternzeitregelungen in den USA nicht nur Gender neutral sind, also Männer und Frauen formal gleich behandeln, sondern ein Familienmodell vor Augen haben, in dem Beschäftigungsauszeiten auch für die Versorgung anderer bedürftiger Familienmitglieder (nicht nur Kinder) genommen werden können (Orloff 2009).

Die Rolle, die Elternzeitregelungen für die Erwerbs- und Karrierechancen von Frauen, speziell von Müttern, spielen, ist in der aktuellen Literatur entsprechend umstritten. Während Elternzeit bis in die 1990er Jahre hinein als Maßnahme galt, um Mütter verstärkt in den Arbeitsmarkt einzubinden, weisen aktuelle Untersuchungen zunehmend auf die negativen Wirkungen langer Erwerbsunterbrechungen für die Erwerbsbeteiligung und 
für den weiteren Karriereverlauf von Frauen und Müttern hin (Ruhm 1998; Misra et al. 2007; Morgan und Zippel 2003; Pettit und Hook 2005). Die USA bieten mit maximal 12 Wochen die kürzesten Auszeiten. Diese sind zudem unbezahlt. Im Vergleich dazu bietet Schweden mit einer Dauer von bis zu 240 Tagen pro Elternteil lange und hoch vergütete Auszeiten für die Kinderbetreuung an. Für 390 Tage werden 80\% des Erwerbseinkommens (bis zu einem Deckelbetrag) gezahlt. Deutschland ermöglicht Eltern nach wie vor Erwerbsunterbrechungszeiten von bis zu drei Jahren pro Kind. Diese Zeiten werden jedoch, selbst nach den neuesten Veränderungen beim Elterngeld, mit einer Kompensationsrate von $67 \%$ des Nettoeinkommens, oder einem Deckelbetrag von maximal $1800 € \mathrm{im}$ ersten Jahr, in geringerem Maße finanziell unterstützt als in Schweden. ${ }^{2}$ Für unsere Fragestellung sind nicht allein die aktuell geltenden Elternzeitregelungen relevant. Vielmehr interessiert uns die Bedeutung der Ausdehnung von Elternzeit für Mütter mit unterschiedlichen Bildungsressourcen. Vor diesem Hintergrund skizzieren wir im Folgenden die Einführung und Entwicklung gesetzlich geschützter Familienzeiten in (West)Deutschland, den USA und Schweden. In Deutschland bestehen Familienzeiten aus zwei Komponenten; erstens dem bereits seit 1952 bestehenden Mutterschutz, der schwangeren Arbeitnehmerinnen das Arbeiten sechs Wochen vor und acht Wochen nach der Geburt untersagt und finanziell durch die Krankenversicherung kompensiert. Das zweite Instrument ist der ehemalige Mutterschaftsurlaub für abhängig beschäftigte Frauen, der erstmalig 1979 eingeführt wurde und damals eine Dauer von vier Monaten hatte. 1986 wurde der Mutterschafts- durch den Erziehungsurlaub und später durch die Elternzeit ersetzt und auch Vätern zugänglich gemacht. Die ursprüngliche maximale Dauer der Elternzeit von 10 Monaten wurde in den folgenden Jahren mehrfach ausgedehnt und beträgt seit 1992 bis zu drei Jahre. Bis 2007 hatten Beschäftigte in Elternzeit Anspruch auf $300 €$ (bzw. 600 DM) Elterngeld monatlich, für die Dauer von bis zu zwei Jahren pro Kind. Gemessen am Durchschnittseinkommen einer Produktionsarbeiterin im Jahre 2000 entspricht dies einem Anteil von lediglich 16\% (Ferrarini 2006). Für Eltern von Kindern, die seit dem 1. Januar 2007 geboren wurden, gelten neue Regelungen, die einen deutlichen Kurswechsel der deutschen Familienpolitik in Richtung Rekommodifizierung und geteilter Sorgearbeit von Männern und Frauen markieren. Die Dauer der finanziellen Kompensation wird auf maximal 14 Monate begrenzt; zwei so genannte Vätermonate eingeschlossen. Diese Neuregelung bietet wesentlich größere finanzielle Anreize als bisher für kürzere Unterbrechungen von bis zu einem Jahr; dies gilt vor allem für die höheren Einkommensschichten, also für hochqualifizierte Frauen (Moss und Wall 2007).

In Schweden wurde die Elternzeit bereits 1974 eingeführt. Damals konnten Väter und Mütter ihre Erwerbstätigkeit bis zu sechs Monate unterbrechen, finanziell kompensiert mit bis zu 90\% ihres Erwerbseinkommens. In den folgenden Jahren wurden die Elternzeitregelungen auf 12 bzw. 15 Monate 1980 und 1989 ausgedehnt. Bereits 1995 wurde der Vatermonat eingeführt, wobei Schweden international eine Vorreiterstellung einnimmt. Das Kompensationsniveau variierte im Laufe der Jahre leicht und liegt seit 1998 bei $80 \%$ des individuellen Bruttolohns. Sowohl in Schweden als auch in Deutschland

2 Diese jüngsten Veränderungen in Deutschland werden in unseren Analysen nicht mit erfasst, da unser Beobachtungsfenster im Jahr 2005 schließt. Für den hier betrachteten Zeitraum ist der Unterschied zwischen Schweden und (West-)Deutschland noch weit größer. 
sind Elternzeiten einer sehr breiten Bevölkerungsschicht zugänglich. In Westdeutschland etwa waren über 90\% der Haushalte, die zwischen 2001 und 2003 ein Kind bekommen haben, anspruchsberechtigt (Dressel et al. 2005).

Im Vergleich zu den meisten OECD-Ländern gibt es in den USA keine bundesstaatenübergreifende gesetzliche Regelung, die Eltern einen Anspruch auf bezahlte Elternzeit garantiert. Eine Studie, die bezahlte Elternschaftsregelungen in 21 ,high income economies" untersucht, zeigt, dass die USA die einzige Nation sind, die keine landesweite finanzielle Unterstützung für Familienauszeiten anbietet (Ray et al. 2009). Im Jahr 1993 wurde in den USA mit dem Family/Medical Leave Act (FMLA) jedoch das erste landesweite gültige Elternzeitgesetz eingeführt. Der FMLA sieht vor, dass Arbeitgeber mit mindestens 50 Beschäftigten, Eltern, die im vorhergehenden Jahr mindestens 1250 Stunden beschäftigt waren, bis zu 12 Wochen unbezahlter Auszeit gewähren. Aufgrund dieser Beschränkungen sind lediglich $45 \%$ der erwerbstätigen Frauen anspruchsberechtigt (Waldfogel 2001). Trotz dieser Einschränkungen hat die Einführung des FMLA in den USA zu einer Ausweitung von Erwerbsunterbrechungen von Müttern geführt (Han und Ruhm 2009). Der Zugang zu FMLA Regelungen ist jedoch stark schichtspezifisch. Eltern in höheren Einkommensschichten haben eher Zugang zu FMLA Regelungen als Eltern in niedrigeren Einkommensschichten (Ray et al. 2009). Neben diesen gesetzlichen Elternzeitregelungen gibt es eine große Varianz von betriebsspezifischen Elternzeitregelungen. Meist handelt es sich um sechswöchige bezahlte Elternschaftsauszeiten. Insgesamt bietet aber nur ein Viertel aller Betriebe in den USA solche bezahlten Elternzeitregelungen an (Ray et al. 2009). Es gibt kaum Studien, welche den Zugang zu diesen unterschiedlichen Regelungen genauer untersuchen. In den wenigen veröffentlichen Untersuchungen zeigt sich, dass der individuelle Zugang zu diesen betriebsspezifischen Regelungen von Elternzeit, ebenso wie der Zugang zu den gesetzlichen Regelungen, schichtabhängig ist. Frauen mit höherer Bildung haben eher Zugang zu bezahlten betrieblichen Elternzeitregelungen als Frauen mit niedrigerer Bildung (Boushey 2008). Einen weiteren Anhaltspunkt für den schichtspezifischen Zugang zu diesen Regelungen liefert eine Untersuchung zu Elternzeitregelungen an Hochschulen in den USA. Yoest (2003, S.2) kommt zu dem Schluss, dass bezahlte Elternzeiten selten seien und vor allem in Elite- und Privatschulen vorkommen. Abgesehen von dem ungleichen Zugang zu diesen Elternzeitregelungen zeigen Klerman und Leibowitz (1994) auch einen generellen Anstieg von Müttern, die bezahlte Elternzeiten nehmen. Dieser Studie zufolge haben 1974 nur 1\%, dagegen 1987 bereits $15 \%$ aller Mütter bezahlte Auszeiten genommen. Zusammenfassend kann festgehalten werden, dass der Zugang zu Elternzeiten in Deutschland und Schweden für abhängig beschäftigte Mütter sehr breit und weitgehend bildungsunspezifisch ist, während in den USA deutliche berufs- und bildungsspezifische Beschränkungen zum Zugang zu staatlich oder betrieblich geschützten Auszeiten existieren.

\section{Stand der Forschung}

Neuere empirische Untersuchungen weisen darauf hin, dass wohlfahrtsstaatliche Regelungen, die eigentlich Ungleichheiten zwischen den Geschlechtern verringern und Frauen beruflich fördern sollten, teilweise das Gegenteil bewirken (Mandel und Semyonov 2005, 
2006; Ruhm 1998). Obwohl Jobgarantien, die normalerweise der zentrale Bestandteil von Elternzeitregelungen sind, nachweislich die Arbeitsmarktbindung von Müttern erhöhen, zeigt der Stand der internationalen Forschung, dass Mütter in Ländern mit großzügigen Elternzeitregelungen seltener hochqualifizierte Berufspositionen erreichen oder halten können (Mandel und Semyonov 2005, 2006). Ob diese Befunde aber auf Mütter aus unterschiedlichen Bildungsschichten gleichermaßen zutreffen und inwiefern die Effekte auf das Vorhandensein und die Form von Elternzeitregelungen in spezifischen Kontexten zurückzuführen sind, ist derzeit unklar. In Schweden scheinen familienbedingte berufliche Auszeiten generell einen nur geringen Einfluss auf das zukünftige Einkommen von Müttern im Vergleich zu kinderlosen Frauen zu haben (Albrecht et al. 1999; Jonsson und Mills 2001). Gleichzeitig bestehen aber deutliche Unterschiede in den Karrierechancen von Frauen im Vergleich zu Männern. Obwohl berufliche Aufwärtsmobilität für schwedische Männer doppelt so wahrscheinlich ist wie für Frauen, scheinen vorherige Phasen in Elternzeit diese Differenz keineswegs zu erklären (Granqvist und Persson 2004). In den USA wurden mutterschaftsbedingte Lohneinbussen von sieben Prozent festgestellt, die jedoch nicht durch entgangene Arbeitsmarkterfahrung erklärt werden können (Budig und England 2001). Ein in der Literatur viel diskutierter Erklärungsmechanismus für die benachteiligte Position von Müttern am Arbeitsmarkt ist der Übergang in Teilzeitarbeit (Budig und England 2001). Im Vergleich mit kinderlosen Frauen sind Mütter - unabhängig vom Länderkontext, deutlich seltener Vollzeit erwerbstätig (Bardasi und Gornick 2000). Dieser Effekt ist am stärksten in Deutschland und am geringsten in den USA. Generell unterscheidet sich die Verbreitung, Definition und Qualität von Teilzeitarbeit in den hier untersuchten Ländern jedoch deutlich voneinander (Kenjoh 2005; O'Reilly und Fagan 1998). So waren im Jahre 2009 38\% der erwerbstätigen Frauen in Deutschland, aber nur 20\% der Frauen in Schweden und 19\% in den USA Teilzeit erwerbstätig (United Nations Statistics Division 2010). Vergleichende Längsschnittuntersuchungen zum Umfang und zur Bedeutung von Teilzeitarbeit bei den Wiedereinstiegen von Müttern sind aufgrund unterschiedlicher Definitionen von Teilzeitarbeit sowie aufgrund meist geringer Fallzahlen bislang selten. Kenjoh (2005) zeigt auf Basis der Daten des soziooekonomischen Panels zum Beispiel, dass drei Jahre nach der Geburt ihres Kindes etwa $30 \%$ der Mütter in Westdeutschland angeben, Teilzeit zu arbeiten. ${ }^{3}$ Für Schweden berichtet Kenjoh einen Anteil von insgesamt etwa $40 \%$ in Teilzeit nach drei Jahren, allerdings basierend auf Angaben zu den tatsächlichen Arbeitsstunden pro Woche. Dieser Anteil teilt sich auf in etwa $10 \%$ der schwedischen Mütter, die so genannte „kurze“ Teilzeitarbeit (1-24 Stunden pro Woche) leisten und etwa 30\%, die in ,langer“" Teilzeit (25-34 Stunden pro Woche) arbeiten (Kenjoh 2005). Eine frühere Längsschnittstudie vergleicht Teilzeitraten für Mütter in Deutschland und in den USA, basierend auf Daten des Soziooekonomischen Panels und des National Survey of Families and Households (Drobnič 2000). Anders als bei Kenjoh (2005) ist in diesem Papier der Übergang zur Elternschaft jedoch nicht Teil des abhängigen Prozesses. Ferner wird nicht deutlich, wie Vollzeit und

3 Ähnliche Ergebnisse zeigen aktuelle Untersuchungen, die sich statt auf Umfragedaten auf neue Registerdatensätze mit sehr hohen Fallzahlen stützen (z. B. Grunow und Müller 2011). Diese Daten befinden sich derzeit allerdings noch in der Testphase und die Studien sind dementsprechend noch nicht abgeschlossen. 
Teilzeit in den Datensätzen definiert werden. Drobnič (2000) berichtet für Frauen fünf Jahre nach Aufnahme der ersten Erwerbstätigkeit ähnliche Teilzeitanteile von 9,9\% (Westdeutschland) bzw. 8,0\% (USA) sowie eine deutlich erhöhte Neigung Teilzeit zu arbeiten für verheiratete Frauen. Ziefle (2004) berichtet auf Basis der Daten des Soziooekonomischen Panels, dass Bruttostundenlöhne in einer qualifizierten Teilzeittätigkeit signifikant um circa 4,5-4,7\% höher liegen als bei vergleichbaren Vollzeittätigkeiten. Dieser Befund wird in neuren Untersuchungen und für andere Länder jedoch nicht nachgewiesen (Gangl und Ziefle 2009). Für die USA berichten aktuelle Längsschnittstudien davon, beinahe den gesamten Verdienstunterschied zwischen Müttern und kinderlosen Frauen („Family wage gap“) durch Kontrolle von Nichterwerbszeiten, Arbeitgeberwechsel nach Erwerbsunterbrechung, Humankapital- und Jobstatusvariablen aufklären zu können (Gangl und Ziefle 2009). Für Deutschland hingegen bleibt ein großer Teil dieser Einkommensdifferenzen unerklärt. Historisch vergleichende Untersuchungen von Frauen verschiedener Geburtskohorten haben gezeigt, dass die Arbeitsmarktbindung von Frauen in Westdeutschland und in den USA stärker geworden ist (Grunow et al. 2006). Erwerbsunterbrechungen finden in beiden Ländern später im Lebenslauf und für kürzere Phasen statt. Die sozialstrukturellen Folgen dieser Entwicklung sind allerdings unklar. In Deutschland scheint die Rückkehr in den Arbeitsmarkt besonders schwierig für Mütter mit kleinen Kindern zu sein (Grunow et al. 2006). Fünf Jahre nach der Aufnahme von Elternzeit sind lediglich $50 \%$ der Frauen in ihren Beruf zurückgekehrt; der größte Teil von ihnen in Teilzeit (Engelbrech 1997; Engelbrech und Jungkunst 2001; Kenjoh 2005). Weitere Längsschnittuntersuchungen deuten einen negativen Zusammenhang zwischen dem Erwerbseinkommen, der relativen beruflichen Position und vorhergehenden Erwerbsunterbrechungen von Müttern an (Aisenbrey et al. 2009; Ziefle 2004).

\section{Erklärungsmechanismen: Unterbrechungsdauer, Institutioneller Kontext und Bildung}

Insgesamt zeigen empirische Befunde einen negativen Einfluss von Erwerbsunterbrechungszeiten auf das Erwerbseinkommen von Frauen und Müttern und zwar trotz der stärkeren Arbeitsmarktbindung und höheren Bildung jüngerer Generationen. Dieser negative Zusammenhang scheint in Deutschland und den USA stärker als in Schweden zu sein. Jungwirth (1999) und Ruhm (1998) argumentieren, dass ausgedehnte Unterbrechungszeiten Kosten für Arbeitgeber verursachen, indem neue Mitarbeiter rekrutiert und für die Erfordernisse des Betriebes ausgebildet werden müssen. Diese Kosten schlagen sich negativ auf die Löhne von Frauen generell nieder (Ruhm 1998; Jungwirth 1999; Datta Gupta et al. 2003). Dieses Argument impliziert, dass die individuellen Kosten vorheriger Erwerbsunterbrechungen für Mütter in Schweden und in Deutschland kleiner sein sollten als in den USA, da in den beiden europäischen Ländern alle Frauen ,pauschal“" mit Lohneinbussen zu rechnen haben, ungeachtet ihres tatsächlichen Verhaltens. Als weiterer zentraler Faktor gilt die so genannte Abwertung des Humankapitals (Mincer und Polachek 1974). Je länger Frauen ihre Erwerbstätigkeit unterbrechen desto stärker werden ihre beruflichen Qualifikationen entwertet und entsprechend größer die Lohneinbußen bei ihrer Rückkehr. Mandel und Semyonov $(2005,2006)$ argumentieren, dass in Ländern 
mit generösen Elternzeitregelungen weniger Frauen in Managementpositionen gelangen als in Ländern mit kurzen oder ohne Elternzeitregelungen. Ein wichtiger Aspekt hierbei sei statistische Diskriminierung, da Arbeitgeber davor zurückschreckten, Managerinnen einzustellen wenn das Risiko hoch sei, diese für ein Jahr oder länger entbehren zu müssen (Mandel und Semyonov 2005, 2006).

In welcher Weise Erwerbsunterbrechungsdauern und Karriereeinbussen in unterschiedlichen Kontexten mit dem Bildungsniveau von Müttern zusammen hängen, ist bislang kaum erforscht. Als gesichert gilt, dass hoch gebildete Frauen und Mütter bessere Berufschancen haben als niedrig qualifizierte. Unklar ist aber, ob dieser Vorteil für Hochqualifizierte am Arbeitsmarkt primär durch Bildungsabschlüsse vermittelt ist oder durch die im Durchschnitt höheren beruflichen Positionen oder durch Unterschiede bei der Vereinbarung von Beruf und Familie, speziell der Erwerbsunterbrechungsdauer. Verschiedentlich wurde argumentiert, aber nicht empirisch getestet, dass längere bezahlte Erziehungszeiten vor allem den Präferenzen gering qualifizierter Frauen in gering entlohnten und unattraktiven Berufen entsprächen, während sich Mittel- und Oberschichtfrauen am Ideal kontinuierlicher Berufstätigkeit orientierten (Morgan und Zippel 2003). Mandel und Shalev (2009b) argumentieren, dass Länder wie Deutschland, die Frauen die Möglichkeit zu langen Erwerbsunterbrechungen mit Jobgarantie geben, ein vorteilhafterer Kontext für Mütter mit niedrigem Bildungsniveau und aus unteren sozialen Schichten, sprich, mit geringerer Karriereorientierung, seien als für Hochqualifizierte. Umgekehrt profitierten hoch qualifizierte Frauen am ehesten in Ländern ohne oder mit nur sehr begrenzten Elternzeitregelungen, zum Beispiel den USA, da in diesen Kontexten der Anreiz zu statistischer Diskriminierung vergleichsweise gering sei (Mandel und Shalev 2009b). Wie oben erläutert ergeben sich in den USA auch unterschiedliche Zugangsraten zu Elternzeitregelungen für Frauen mit unterschiedlichen Bildungsniveaus (Boushey 2008; Ray et al. 2009). Dies impliziert zum einen, dass Frauen in Abhängigkeit des institutionellen Kontextes unterschiedliche Entscheidungen hinsichtlich der Dauer ihrer Erwerbsunterbrechungen treffen. Zum anderen kann angenommen werden, dass sich bei den Erwerbsunterbrechungsdauern selbst sowie bei den beruflichen Folgen von Erwerbsunterbrechungen innerhalb eines Landes Unterschiede zwischen Müttern mit hohem und niedrigem Bildungsniveau finden lassen; teilweise vermittelt über deren berufliche Position. Gerade diese Unterschiede sollten gesetzliche Elternzeitregelungen jedoch ausgleichen. Für unsere Analysen ergibt sich daraus, dass zum einen die Dauer der Erwerbsunterbrechungen, zum anderen die Qualität der Wiedereinstiege von Müttern zentrale Größen sind, die mit Blick auf die jeweiligen Bildungseffekte aufeinander bezogen interpretiert werden müssen.

\section{Hypothesen}

Auf Basis des Forschungsstandes und vor dem Hintergrund der institutionellen Rahmenbedingungen in Deutschland, Schweden und den USA gehen wir davon aus, dass Mütter in Deutschland die längsten Erwerbsunterbrechungen haben und Mütter in den USA die kürzesten (Aisenbrey et al. 2009). Darüber hinaus erwarten wir folgende empirische Ergebnisse: 
H1: Je höher die Bildung umso schneller kehren Mütter nach der Geburt eines Kindes wieder in den Arbeitsmarkt zurück. Dieser Effekt sollte in den drei Ländern unterschiedliche Rückkehrmuster produzieren.

Dieser Hypothese zufolge gilt ganz generell: Je höher die Bildung umso mehr gleicht das Berufsleben einer Karriere, die berufliche Abstiegsrisiken oder Aufstiegschancen bereithält. Frauen mit höherer Bildung haben somit allgemein „mehr zu verlieren“ und „mehr zu gewinnen" als Frauen mit niedrigerer Bildung. Daher erwarten wir, dass gut ausgebildete Frauen schneller wieder in das Arbeitsleben zurückkehren. In den USA erwarten wir hier die stärksten Differenzierungen bei der Frage, ob nach der Geburt überhaupt eine Erwerbsunterbrechung stattfindet, da hier die kontinuierliche Erwerbstätigkeit die Norm ist. Entsprechend sollte die Sorge, dem Arbeitgeber durch eine Auszeit eine geringe Karriereorientierung zu signalisieren, besonders unter gut ausgebildeten Müttern hoch sein. Dagegen sollten Frauen, die sich durch umfassende staatliche Elternschaftsregelungen wie in Schweden und Deutschland ,geschützt“ fühlen, von diesem Effekt weniger stark betroffen sein. Um analytisch die Bildungseffekte von den primär beruflich vermittelten Effekten zu trennen, zeigen wir neben deskriptiven Survivorkurven multivariate Modelle zum beruflichen Wiedereinstieg sowohl mit als auch ohne Kontrolle von sozioökonomischem Prestige.

H2: Da in allen drei Ländern der Anspruch auf Elternzeit ausgeweitet wurde, erwarten wir längere Unterbrechungen nach der Geburt eines in den späteren Perioden geborenen Kindes als in früheren Phasen.

Die in Abschn. 2 diskutierten länderspezifischen Merkmale legen nahe, dass sich für Deutschland die Veränderungen über den Zeitraum der ersten drei Jahre nach der Geburt konzentrieren. Für Schweden erwarten wir geringere Veränderungen, die vor allem im Bereich zwischen zwölf und 15 Monaten liegen sollten. Für die USA erwarten wir stattdessen nach 1992, dem Zeitpunkt des Inkrafttretens des FMLA, einen Anstieg derer, die ihre Erwerbstätigkeit überhaupt unterbrechen. Es ist jedoch einschränkend zu berücksichtigen, dass in den USA nur eine Minderheit erwerbstätiger Mütter Anspruch auf die im FMLA vorgesehenen drei Monate unbezahlte Elternzeit hat. Für die Unterbrechungsdauer nach der Geburt des ersten Kindes, d.h. ungeachtet der Geburt weiterer Kinder, fanden Aisenbrey et al. (2009) empirisch keinen eindeutigen Hinweis darauf, dass die tatsächlichen Unterbrechungsdauern den gesetzlichen Ausweitungen folgen. In dem vorliegenden Beitrag erweitern wir die Analysen um eine Multi-Episoden-Betrachtung, bei der der Prozess erstens bei der Geburt eines weiteren Kindes rechts zensiert wird und zweitens das Prozessfenster erneut für zweit-, dritt- und viert- geborene Kinder eröffnet wird. Dies hat den methodischen Vorteil, dass für alle Geburten die gleiche jeweils politikrelevante Phase verglichen wird. Insofern dient diese Untersuchung auch der Validierung von Aisenbrey et al. (2009), in der der abhängige Prozess bei der Geburt weiterer Kinder nicht rechts zensiert wurde.

H3: Zugangshypothese. Der schichtspezifische Zugang zu Elternzeitregelungen in den USA führt zu mehr beruflicher Stabilität bei den Wiedereinstiegen für Mütter mit höherem Bildungsniveau; dagegen wirkt die Inanspruchnahme von Elternzeit in Deutschland und Schweden bezogen auf die Rückkehrposition bildungsneutral. 
Wenn Elternzeitregelungen wie in Deutschland und Schweden relativ inklusiv sind, dann sollten sie allen abhängig beschäftigten Müttern, unabhängig von deren Bildungsniveau und sozioökonomischen Status, ermöglichen, nach der Geburt ihres Kindes innerhalb der gesetzlichen Frist von Elternzeitregelungen Gebrauch zu machen und auf eine gleichwertige berufliche Position zurückzukehren. In den USA erwarten wir dagegen, dass vor allem Mütter mit höherer Bildung Zugang zu den gesetzlichen und organisationsspezifischen Unterbrechungszeiten haben und deshalb nach einer Unterbrechung beruflich stabiler sind als ihre geringer qualifizierten Peers. Zum Test dieser Hypothese berechnen wir ein Modell für die Qualität der Rückkehrposition (gleich, besser oder schlechter als vor der Geburt des Kindes), bei dem die Effekte für das Bildungsniveau der Mütter abgebildet werden.

H4: Regime-Klassen-Hypothese. Die Vor- und Nachteile der unterschiedlichen Regime wirken mit Blick auf die Karrierekontinuität bildungsschichtspezifisch. In Schweden und Deutschland profitieren die Hochqualifizierten durch die Ausdehnung geschützter Elternzeiten am wenigsten. In den USA profitieren die Hochqualifizierten am meisten.

Diese Hypothese greift die aktuelle Diskussion um die klassenspezifischen Karrierekonsequenzen für Frauen in unterschiedlichen wohlfahrtsstaatlichen Kontexten auf. Wir übertragen hier das Argument, das Mandel und Shalev (2009b) allgemein für Frauen unterschiedlicher sozialer Klassen in konservativen, sozialdemokratischen und liberalen Regimes formuliert haben, auf die konkreten Kontexte und Bedingungen für Mütter in Deutschland, Schweden und den USA. Dem Argument von Mandel und Shalev (2009b) folgend erwarten wir, dass hoch qualifizierte Mütter in Deutschland und Schweden mit Blick auf ihre berufliche Rückkehrposition am wenigsten von den Elternzeitausdehnungen profitieren. Das liegt der Hypothese zufolge daran, dass vor allem die gering qualifizierten Mütter mit vergleichsweise unattraktiven Berufen oder geringer Erwerbsorientierung die Pufferwirkung des verlängerten beruflichen Statuserhalts in Anspruch nehmen und erfahren. D.h., wir erwarten, dass sich die Bedingungen für diese gering qualifizierte Gruppe relativ zu den Hochqualifizierten verbessern. In den USA erwarten wir hingegen, dass vor allem die Hochqualifizierten von Elternzeitregelungen profitieren (Mandel und Shalev 2009b; Boushey 2008). Dieser Vorteil wird durch den privilegierten Zugang für Hochqualifizierte zu diesen Regelungen vermittelt. Wir testen diese Hypothese durch ein Interaktionsmodell für Wiedereinstiege auf dem gleichen, höheren und niedrigeren beruflichem Prestigeniveau relativ zur beruflichen Position vor der Geburt des Kindes. Dabei wird das Bildungsniveau der Mütter mit den historischen Perioden, in denen die gesetzlichen Ausdehnungen stattfanden, direkt in Beziehung gesetzt. So zeigt sich, in welchen Ländern die Ausdehnung, oder in den USA die Einführung, landesweiter gesetzlich geschützter Auszeiten eventuell bestehende Bildungsunterschiede bei der beruflichen Kontinuität von Müttern verringert oder weiter verstärkt. 


\section{Daten}

Die Analysen beruhen auf länderspezifischen Längsschnittdaten, die den Zeitraum seit den 1970er Jahren abdecken und die für unsere Untersuchungen in einem aufwendigen Datenaufbereitungsprozess harmonisiert wurden. Die länderspezifisch abgebildeten Zeiträume überlappen für den gesamten Zeitraum der 1980er und 1990er Jahre. Für Deutschland beobachten wir den Zeitraum von 1972 bis 2005, für Schweden 1969 bis 2000, und für die USA 1980 bis 2000. In allen drei Ländern fällt die große Mehrheit der beobachteten Geburten in die 1980er und 1990er Jahre. Mit Hilfe ereignisanalytischer Verfahren untersuchen wir, wie lange Mütter nach der Geburt eines Kindes ihre Erwerbstätigkeit unterbrechen und ob der Wiedereinstieg, so wie in Elternzeitprogrammen vorgesehen, auf dem vorherigen beruflichen Prestigeniveau erfolgt.

Für die USA nutzen wir die Daten des National Longitudinal Survey of Youth (NLSY) (dokumentiert in Bureau of Labor Statistics 2004). Die NLSY Daten basieren auf einer national repräsentativen Stichprobe von 12686 Männern und Frauen, die zwischen 1957 und 1964 geboren wurden und die im Abstand von zwei Jahren wiederbefragt werden. Für Deutschland nutzen wir den westdeutschen Teil der Deutschen Lebensverlaufstudie (GLHS-West), die unter der Leitung von Karl Ulrich Mayer am Berliner Max-PlanckInstitut für Bildungsforschung erhoben wurde (Mayer 2008). Die West-Stichproben dieses Datensatzes bestehen aus zum Zeitpunkt der Stichprobenziehung in Westdeutschland lebenden Mitgliedern der Geburtskohorten 1954-1956, 1964 und 1971. ${ }^{4}$ Für diese Kohorten können wir die berufliche Entwicklung bis zum Alter von 34 bzw. 35 Jahren verfolgen. Diese Einschränkung unseres Beobachtungsfensters führt dazu, dass wir für Deutschland Wiedereinstiege vor allem für jene Frauen beobachten, die Kinder in ihren Zwanzigern bekommen haben. Unsere Ergebnisse lassen sich nicht ohne weiteres auf andere Kohorten übertragen, was bei der Interpretation zu berücksichtigen ist. Sie fügen sich jedoch widerspruchslos in die Ergebnisse früherer Studien, die auf Grundlage des Sozio-oekonomischen Panels (SOEP) gewonnen wurden und ein weiteres Kohorten- und Altersspektrum abdecken, jedoch andere Limitationen aufweisen (Ziefle 2004; Kenjoh 2005). ${ }^{5}$ Für Schweden nutzen wir die Daten des Swedish Level of Living Survey (LNU) von 1991 und 2000, einer Panelstudie. Die Daten des LNU basieren auf einer Zufallsstichprobe von 1/1000 der schwedischen Bevölkerung zwischen 18 und 75 Jahren. Da das Erhebungsverfahren der LNU in Anlehnung an die deutsche Lebensverlaufstudie entwickelt wurde, eignen sich beide Datensätze besonders für den Ländervergleich.

4 In den beiden jüngsten Kohorten wurden erstmalig auch Deutschsprachige nicht-deutscher Staatsangehörigkeit interviewt. Für die ältere Kohorte sind nur deutsche Staatsbürger einbezogen. Nähere Informationen zu den einzelnen Studien sind unter http://www.yale.edu/ciqle/ GLHS/index.html zu finden.

5 Der einzige alternative Längsschnittdatensatz für Deutschland für diesen Ländervergleich wäre das SOEP gewesen. Der große Nachteil des SOEP gegenüber der GLHS für unsere Fragestellung besteht jedoch darin, dass die Paneldaten erst seit 1984 erhoben wurden und keine ausreichenden Daten für den historisch so wichtigen Zeitraum vor Einführung des Erziehungsurlaubs vorliegen. Diese Einschränkung hätte auch die historische Passung mit den schwedischen und US-Daten verschlechtert. 
Da unser Augenmerk auf den Konsequenzen von Erwerbsunterbrechungen liegt, beziehen wir nur Frauen in die Analysen ein, die zumindest einen von sieben Monaten vor der Geburt ihres ersten Kindes erwerbstätig waren. Damit selektieren wir Frauen die schneller in den Beruf zurückkehren als jene, die während ihrer Schwangerschaft überhaupt nicht erwerbstätig waren (Berger und Waldfogel 2004; Hofferth und Curtin 2006). Wir schließen auch selbständige Frauen aus den Analysen aus, da diese keinen gesetzlichen Anspruch auf Elternzeit haben. Diesen Selektionskriterien folgend enthält unser Analysedatensatz für die USA 3531 Frauen, geboren zwischen 1957 und 1964. Der deutsche Analysedatensatz besteht aus 963 Frauen der Geburtskohorten 1954-1956, 1964 und 1971. Der Datensatz für Schweden enthält 718 Frauen, die zwischen 1950 und 1975 geboren wurden.

\section{Abhängige Prozesse}

Wir modellieren Erwerbsunterbrechungen von Müttern aus der Perspektive von Elternzeitregelungen; diese gelten pro Kind und sehen nach einer Unterbrechung die Rückkehr in den gleichen (oder einen gleichwertigen) Job beim selben Arbeitgeber vor. Entsprechend beginnt der abhängige Prozess mit der Geburt eines Kindes und wird bei Geburt eines weiteren Kindes rechts zensiert, wenn vorher keine Rückkehr in den Beruf stattgefunden hat. Bei jeder weiteren Geburt beginnt die Prozesszeit von neuem. Manche Frauen, speziell in den USA, unterbrechen ihre Erwerbstätigkeit überhaupt nicht. Diesen Frauen rechnen wir eine künstliche Unterbrechungszeit von zwei Wochen an. Wir zeigen zunächst Survivorkurven für die Unterbrechungsdauer getrennt für unterschiedliche Bildungsabschlüsse der Mütter gemäß Hypothese 1. Die Frage ist hier, ob Mütter mit hohem Schulbildungsniveau schneller in den Beruf zurückkehren. In einer zweiten Abbildung nähern wir uns zunächst deskriptiv der zweiten Hypothese an, derzufolge die Ausdehnung des gesetzlichen Elternzeitanspruchs in Deutschland und Schweden, oder die Einführung des FMLA in den USA, tatsächlich einen sichtbaren Einfluss auf die Länge der Erwerbsunterbrechungen von Müttern hat. Die unterschiedenen Zeiträume richten sich nach den wichtigsten gesetzlichen Veränderungen bei den Elternzeitregelungen. Für Deutschland vergleichen wir den Zeitraum vor 1986, mit kurzem Mutterschaftsurlaub, 1986 bis 1991, nach der Einführung der Erziehungs- oder Elternzeit, und den Zeitraum nach 1991, nach maximaler Ausdehnung der Elternzeit. Für die USA unterscheiden wir die Perioden vor 1987, 1987 bis 1992, und nach 1992, den Zeitraum nach Einführung des FMLA. Für Schweden unterscheiden wir den Zeitraum bis 1979, als Elternzeit auf 180 Tage ausgedehnt wurde, den Zeitraum von 1980 bis 1988, als eine Ausdehnung auf 360 Tage stattfand und den Zeitraum seit 1989, nach einer erneuten Ausdehnung auf 450 Tage. Wir berechnen drei multivariate Cox-Regressionsmodelle zur Erwerbsunterbrechungsdauer von Müttern.

In einem zweiten Schritt betrachten wir berufliche Auf- und Abstiege sowie Rückkehr auf demselben beruflichen Niveau als konkurrierende Ereignisse, gemessen als Veränderung des beruflichen SIOPS-Prestige-Wertes vor und nach der Geburt eines (weiteren) Kindes (Treiman 1977). Diese Analyse dient der Untersuchung, inwiefern das von Mandel und Shalev (2009b) vorgetragene Argument, die Vor- und Nachteile unterschied- 
licher Regimes und Politiken wirkten schichtspezifisch (Hypothese 4), auf unsere Daten zutrifft.

Um die Vergleichbarkeit der Erwerbsunterbrechungen von Müttern in den verschiedenen historischen Perioden zu gewährleisten, wurde das Untersuchungsfenster in allen Analysen acht Jahre nach Geburt des jeweils jüngsten Kindes rechts zensiert. Diese Entscheidung trägt auch dem Umstand Rechnung, dass sowohl in den schwedischen als auch in deutschen Daten nach acht Jahren die Risikopopulation zu gering ist, um robuste Schätzungen zu liefern. Aufgrund der begrenzten Fallzahlen unterscheiden wir nicht zwischen Rückkehrmustern in Vollzeit oder Teilzeit. Es ist aber davon auszugehen, dass Wiedereinstiege in Deutschland und Schweden zum großen Teil in Teilzeit erfolgen, während Mütter in den USA vornehmlich vollzeiterwerbstätig bleiben (Bardasi und Gornick 2008).

\section{Unabhängige Variablen}

Bildung. Die Bildungsvariablen, auf denen das Hauptaugenmerk unserer Analysen liegt, sind kontext- oder länderspezifisch operationalisiert, da die Bildungssysteme in den drei Ländern sehr unterschiedlich sind. Wir haben die Kategorien so gewählt, dass die Jahre, die Personen im allgemeinen Schulbildungssystem verbringen, dennoch möglichst vergleichbar sind. Für die USA umfassen die Bildungsvariablen die Kategorien „keinen High School Abschluss“, „High School Abschluss“ und „mindestens ein Jahr College“. In Deutschland unterscheiden wir zwischen „,maximal Hauptschulabschluss“, „Mittlerer Reife“ und „(Fach-)Abitur“. Für Schweden wird in der niedrigsten Bildungskategorie der Abschluss nach 9 Schuljahren, „Grundskola“, erfasst, die mittlere Kategorie umfasst den Abschluss nach elf bis zwölf Jahren, „Gymnasieskola“, und die höchste Kategorie „Högskola" fasst Personen zusammen, die über 12 Jahre im Schulbildungssystem verbracht haben.

Berufsprestige. Das Berufsprestige, gemessen in Form des SIOPS-Codes zum Zeitpunkt unmittelbar vor der Geburt des Kindes, geht als zweiter Indikator in einen Teil der Analysen ein. Speziell interessiert uns, wie sich die Bildungseffekte verändern, wenn für das Prestige kontrolliert wird, gegenüber dem Modell ohne Prestige. Diese Unterscheidung ist wichtig, um den unmittelbar berufsbezogenen Einfluss auf die Erwerbsunterbrechungsdauer von den generellen Bildungseffekten zu trennen.

Geburtsperiode des Kindes. Die Geburtsperiode des Kindes beziehen wir in Form von drei Dummy Variablen ein. Die Einteilung dieser Perioden orientiert sich an Zeitpunkten zu denen, wie im vorhergehenden Abschnitt erläutert, einschneidende familienpolitische Veränderungen stattgefunden haben.

Arbeitsmarkterfahrung und Unterbrechungen, gemessen in Jahren. Wir verwenden die kumulative Erwerbszeit zum Zeitpunkt der Geburt des Kindes als Indikator für Arbeitsmarkterfahrung. Erwerbsunterbrechungen werden in den drei Ländern unterschiedlich gemessen. In den USA akkumulieren wir Erwerbsunterbrechung. In Deutschland und Schweden sind dagegen in dieser Variable Zeiten in Elternzeit und familienbedingte Nichterwerbstätigkeit zusammengefasst. 
Familienstrukturen. Der Effekt von Familienstrukturen auf den Wiedereintritt in den Arbeitsmarkt wird mit Dummy Variablen gemessen: Ein Kind, zwei Kinder, mehr als zwei Kinder, und Partner im Haushalt ja/nein.

Hintergrundinformationen. Als Hintergrundvariablen haben wir das Alter der Frauen bei Geburt des Kindes in die Modelle aufgenommen. Aufgrund unterschiedlicher struktureller Chancen verschiedener Bevölkerungsgruppen kontrollieren wir in den USA auch, ob die Mütter afroamerikanischer Abstammung sind.

\section{Ergebnisse}

Abbildung 1 zeigt die Survivorkurven für die Rückkehr zum Arbeitsplatz nach der Geburt eines Kindes. Gemäß unserer ersten Hypothese unterscheiden wir in dieser Darstellung drei unterschiedliche Gruppen von Müttern: jene mit niedrigem, mittlerem und hohem Schulbildungsniveau. Ein Viertel der Mütter in Deutschland mit Abitur kehren direkt nach dem Mutterschutz an den Arbeitsplatz zurück, während der Anteil der Mütter mit mittlerer Reife und maximal Hauptschulabschluss mit 15\% deutlich niedriger liegt. Nach Ablauf der gesetzlich geschützten, maximal möglichen Erwerbsunterbrechungszeit von 3 Jahren haben sich die Rückkehrraten der mittel und hoch qualifizierten Mütter einander angeglichen. Die Schere zwischen den Müttern mit geringer und denen mit mittlerer oder hoher Schulbildung wird deutlich größer.

Schweden markiert hier das andere Extrem. Nahezu alle schwedischen Mütter unterbrechen ihre Erwerbstätigkeit nach der Geburt eines Kindes für circa ein halbes Jahr; und zwar unabhängig von ihrem Bildungsniveau. Bildungsspezifische Differenzen ergeben sich erst nach Ablauf der Elternzeit und sind besonders ausgeprägt nach zwei Jahren. $\mathrm{Zu}$ diesem Zeitpunkt sind $86 \%$ der Mütter mit Universitätshintergrund wieder zurück am Arbeitsplatz. Für die Frauen mit mittlerem Schulbildungsniveau liegt der Prozentsatz etwas niedriger bei $82 \%$. Für die niedrigste Qualifikationsgruppe liegt der Prozentsatz mit $67 \%$ fast $20 \%$ niedriger als bei den hoch ausgebildeten Frauen.

In den USA zeigen sich Bildungsunterschiede bereits bei der Frage, welche Mütter überhaupt nach der Geburt ihre Erwerbstätigkeit unterbrechen. Vierzig Prozent aller Mütter, die ein College besucht haben, aber nur weniger als ein Viertel der Highschool Abbrecherinnen, bleiben kontinuierlich erwerbstätig. Nach drei Monaten, der maximal geschützten Auszeit für amerikanische Mütter, sind 45\% der High School Abbrecherinnen, aber über $60 \%$ der Frauen mit Collegeabschluss zurück im Job. Im Gegensatz zu diesen relativ kurzen Unterbrechungen dreht sich das Bild jedoch noch vor dem Ablauf von zwei Jahren um. Nach fünf Jahren sind deutlich mehr Frauen mit niedrigen Bildungsabschlüssen (90\%) in den Arbeitsmarkt zurückgekehrt als Frauen mit höheren Bildungsabschlüssen (82\%). Am Ende unseres Beobachtungsfensters hat sich diese Schere noch weiter geöffnet: nur noch 5\% aller Mütter mit niedrigem Bildungsabschluss sind nicht in den Arbeitsmarkt zurückgekehrt, wohingegen 15\% aller Mütter mit einem Collegeabschluss nicht zurückgekehrt sind. Möglicherweise spiegelt die „Umkehrung“ des Bildungseffektes in den USA Klasseneffekte wider, indem sowohl berufliche Kontinuität als auch lange Sorgezeiten etwas sind, das man sich leisten können muss. 


\begin{tabular}{|l|}
\hline West- \\
Deutschland \\
- maximal \\
Hauptschul- \\
abschluss \\
- - Mittlere Reife \\
… (Fach-)Hoch- \\
schulreife \\
\hline
\end{tabular}

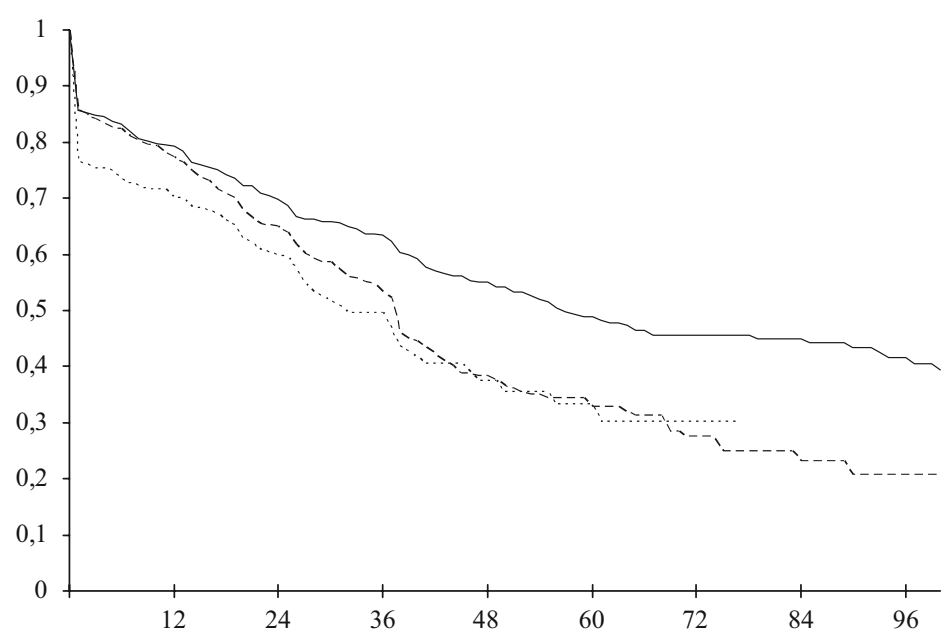

\begin{tabular}{|l|}
\hline Schweden \\
— Schulab- \\
schluss nach \\
neun Jahren; \\
Grundskola \\
- - Schulab- \\
schluss nach \\
11-12 Jahren; \\
Gymnasieskola \\
mindestens \\
ein Jahr \\
Universität; \\
Högskola
\end{tabular}
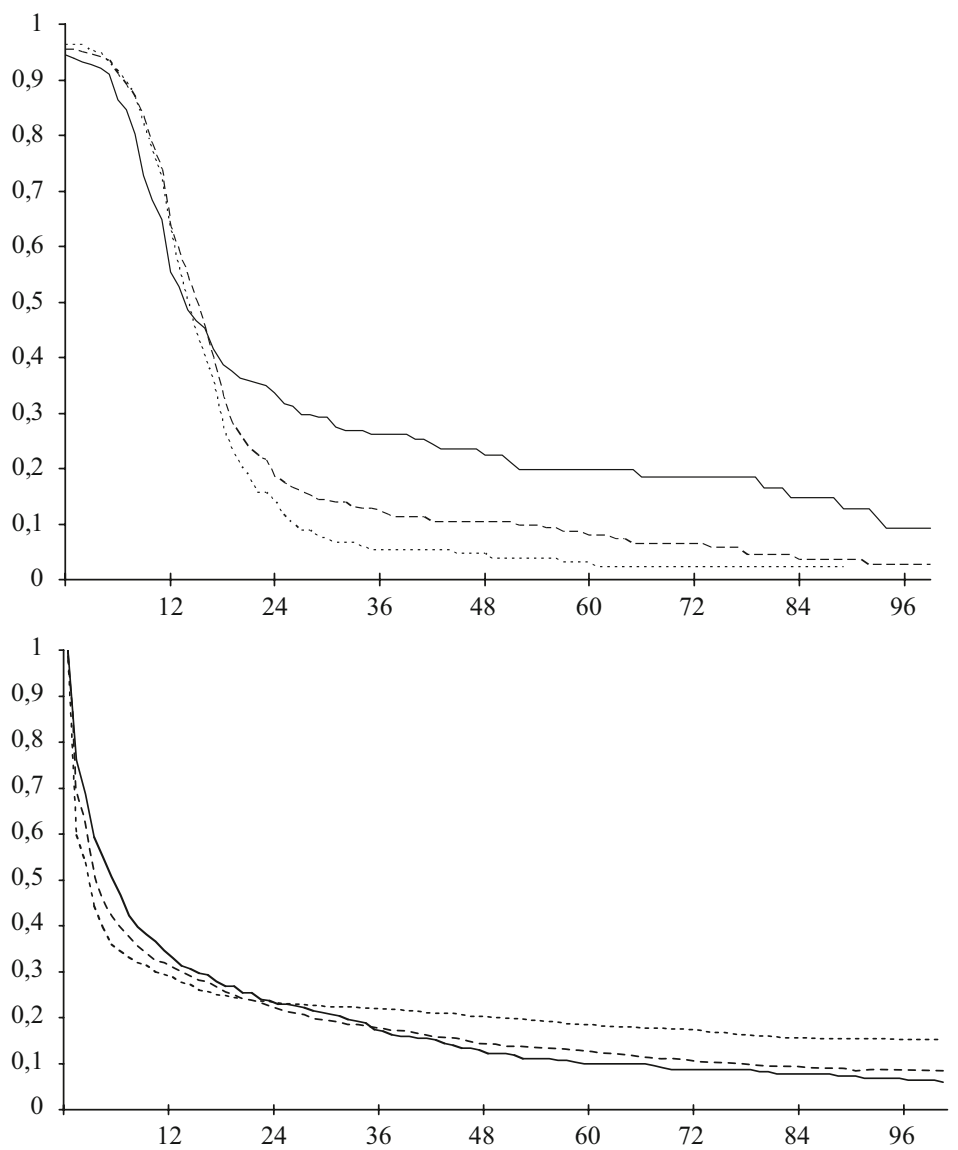

Abb. 1: Dauer der Erwerbsunterbrechung von Müttern nach Geburt eines Kindes, differenziert nach Schulbildungsniveau, Dauer in Monaten, Kaplan-Meier Schätzung, Multiepisodenmodell, rechtszensiert bei der Geburt weiterer Kinder. (Quelle: GLHS-West Kohorten 1954-1956, 1964, 1971, NLSY Kohorten 1957-1964, LNU Kohorten 1950-1975, eigene Berechnungen) 
Gemäß unserer zweiten Hypothese zeigen wir in Abb. 2 die Dauer der Erwerbsunterbrechungen in verschiedenen historischen Phasen. In Deutschland tendieren Mütter zunächst dazu, die Ausweitung der Elternzeit zu nutzen. Mütter, deren erstes Kind zwischen 1987
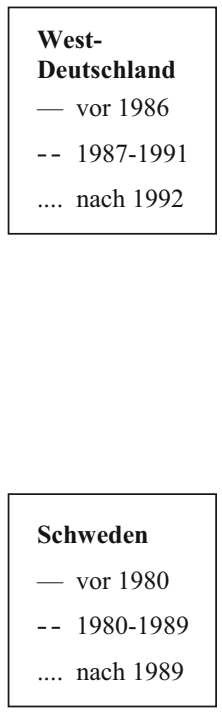
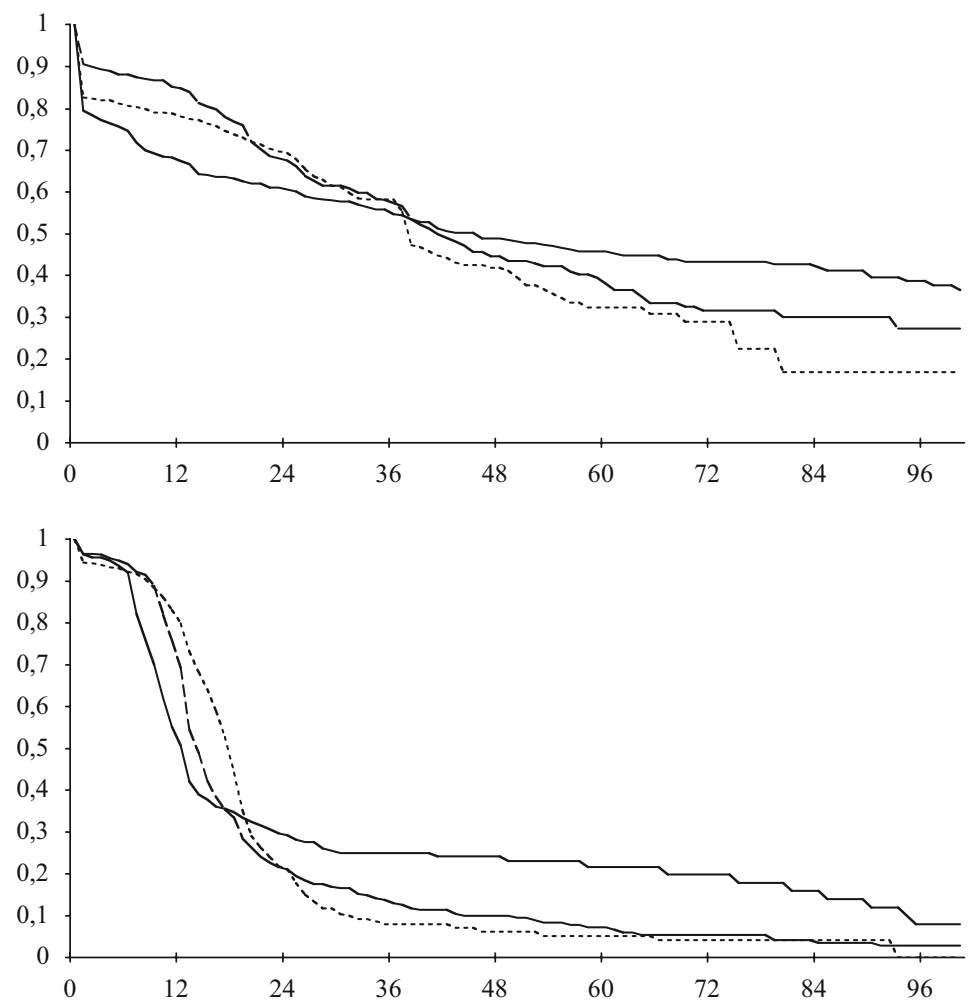

\section{USA}

- vor 1987

- 1987-1992

.... nach 1992

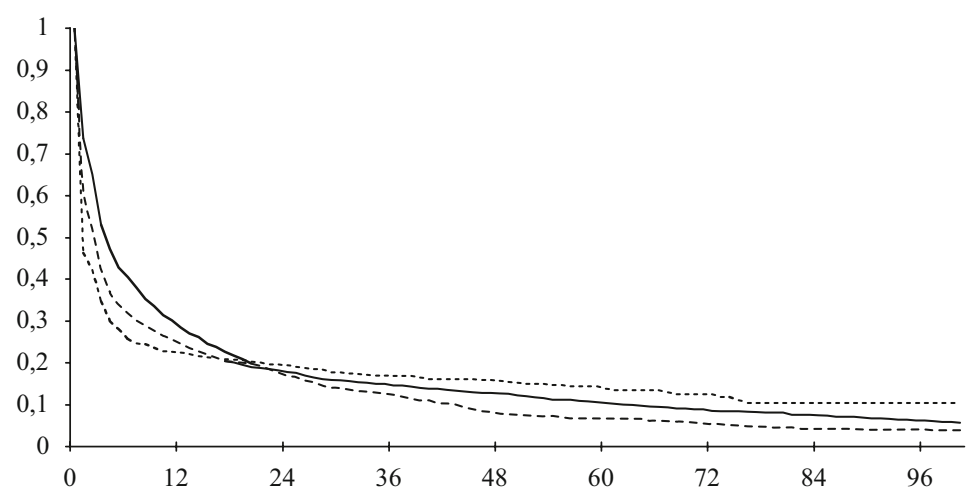

Abb. 2: Dauer der Erwerbsunterbrechung von Müttern nach Geburt des ersten Kindes, differenziert nach Perioden, Dauer in Monaten, Kaplan-Meier Schätzung, rechtszensiert bei Geburt weiterer Kinder. (Quelle: GLHS-West Kohorten 1954-1956, 1964, 1971, NLSY Kohorten 1957-1964, LNU Kohorten 1950-1975, eigene Berechnungen) 
und 1992 geboren wurde, unterbrechen ihre Erwerbstätigkeit für längere Zeit als Mütter, die ihr Kind in der ersten Hälfte der 1980er Jahre oder früher hatten. Die jüngste Mutterschaftskohorte scheint demgegenüber in zwei größere Gruppen gespalten zu sein: jene, die direkt nach der Mutterschutzzeit an den Arbeitsplatz zurückkehren (18\%) und jene, die jeweils die maximal verfügbare Elternzeit ausschöpfen. Die Kurve für die jüngste Kohorte fällt kurz vor Vollendung der Drei-Jahres Grenze, der maximal möglichen Elternzeitdauer, deutlich sichtbar um mehr als $10 \%$ ab. Danach unterscheiden sich die Kurven nicht länger statistisch signifikant voneinander, wie zusätzliche Analysen gezeigt haben. Auch für Schweden sehen wir für den Zeitraum der Ausweitung der Elternzeit auf zwölf und später auf 15 Monate eine zunächst geringere Rückkehrneigung von Müttern. ${ }^{6}$ Auch dieses Muster entspricht unseren Erwartungen (Hypothese 2). Interessant ist, dass sich trotz dieser tendenziell längeren Erwerbsunterbrechungen in den ersten eineinhalb Jahren nach der Geburt eines Kindes längerfristig die Rückkehrraten in einen gegenteiligen Periodentrend umkehren: Während bei den vor 1980 geborenen Kindern der Anteil der Rückkehrerinnen nach circa zwei Jahren bei etwa 20-25\% nahezu stagniert, ist die Arbeitsmarktbindung von Müttern bei späteren Geburten insgesamt deutlich, d.h. um etwa $10 \%$, höher. Zeitgleich mit der Ausweitung der Elternzeitprogramme hat die Arbeitsmarktbindung schwedischer Mütter also zugenommen. Für die USA zeigt sich, dass die Einführung des FMLA 1992 im Durchschnitt nicht zu einer Ausweitung von Erwerbsunterbrechungen amerikanischer Mütter geführt hat. Stattdessen kehren Mütter in jüngerer Zeit durchschnittlich immer schneller an ihren Arbeitsplatz zurück und der Anteil derer, die ihre Erwerbstätigkeit überhaupt unterbrechen, ist gesunken. Damit wird unsere Hypothese, die zeitliche Ausdehnung des gesetzlichen Anspruchs auf Elternzeit führe per se zu längeren Erwerbsunterbrechungszeiten pro Kind, nur für Schweden und Deutschland bestätigt. Dieses Ergebnis nuanciert die Befunde von Aisenbrey et al. (2009), die aufgrund eines anderen Forschungsdesigns die gesetzlichen Ausweitungseffekte für Schweden nicht finden konnten.

Zusammenfassend sprechen diese ersten deskriptiven Ergebnisse dafür, dass sich für Mütter im us-amerikanischen und deutschen Länderkontext zunächst die Frage stellt, ob die Erwerbstätigkeit überhaupt unterbrochen wird. In Schweden ist dies nicht der Fall. Sowohl für Deutschland als auch für die USA finden wir hier, trotz der für den deutschen Kontext eher kruden Messung von Bildung, deutliche Unterschiede, nach denen hochqualifizierte Mütter seltener eine Erwerbsunterbrechung nach der Geburt eines Kindes haben. Erst in einem zweiten Schritt wird die Frage der Unterbrechungsdauer relevant. Letzteres ist auch in Schweden der Fall, wo praktisch alle Mütter nach der Geburt ihres Kindes ihre berufliche Tätigkeit unterbrechen. Wie erwartet finden wir Hinweise auf bildungsspezifische Unterbrechungsmuster in allen drei Ländern. Dem Zusammenhang von Unterbrechungsdauer, Bildung und beruflicher Kontinuität widmen wir uns im nächsten Analyseschritt.

Tabelle 1 zeigt für alle drei Länder jeweils drei verschiedene multivariate Cox-Modelle zur Erwerbsunterbrechungsdauer. Von besonderem Interesse sind die Bildungseffekte, die in Modell 1 jeweils nach Kontrolle der Berufsposition der Mutter zum Zeitpunkt der

6 Diese Unterschiede sind für die politikrelevanten Zeiträume jeweils statistisch signifikant auf dem $5 \%$-Niveau. 


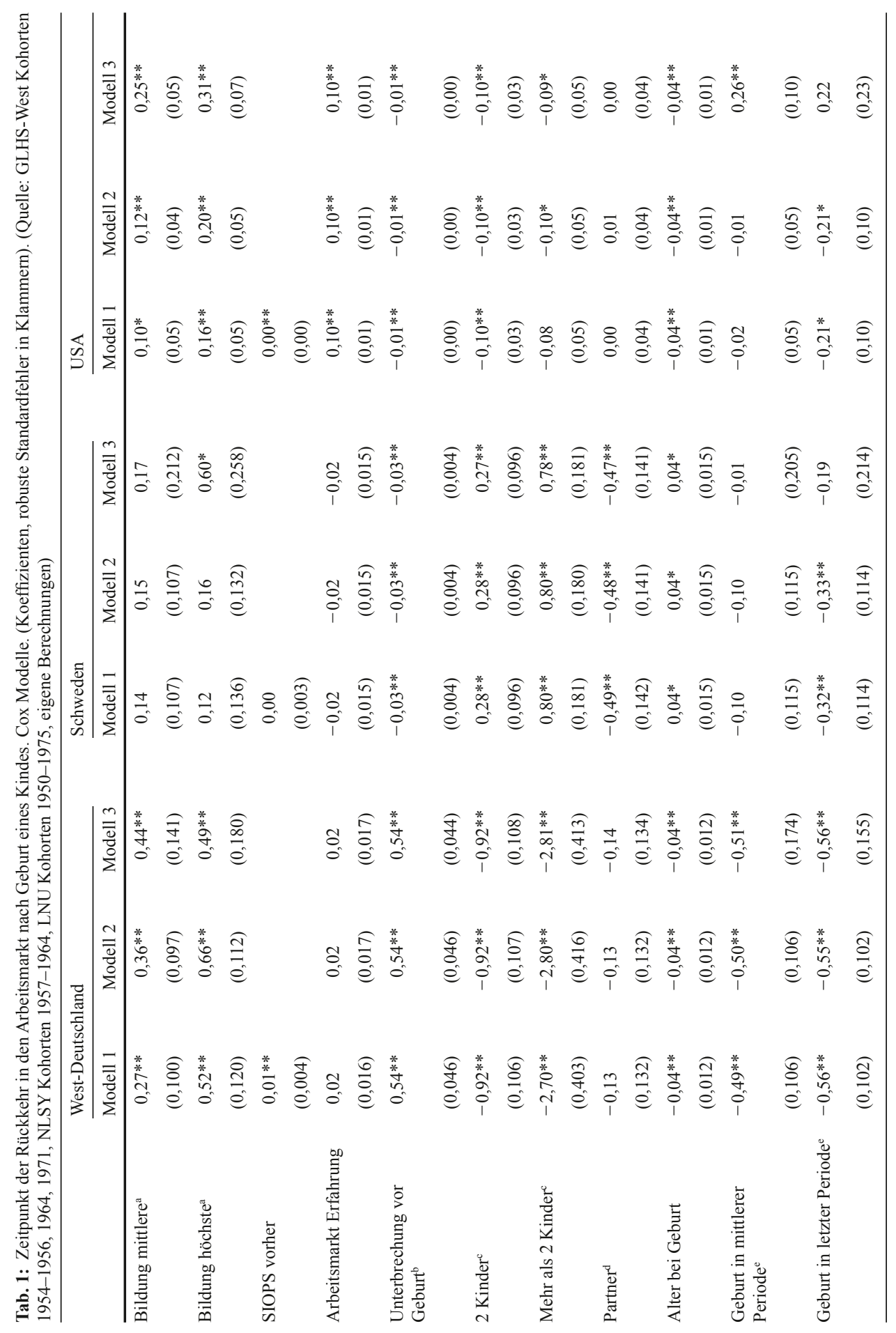




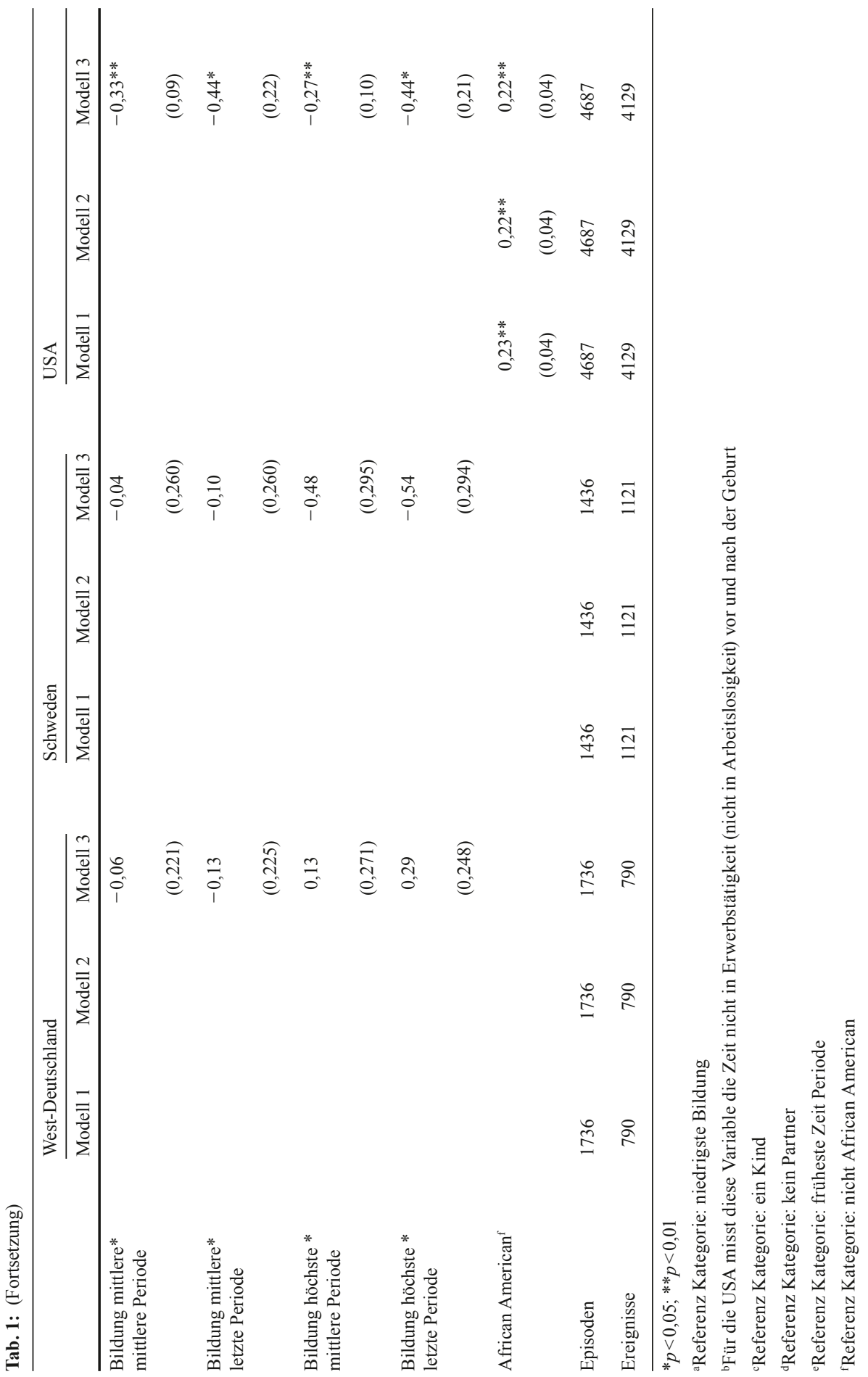


Geburt des Kindes, in Modell 2 ohne diese Kontrollvariable in die Berechnungen eingehen. Die Tabellen zeigen Koeffizienten, keine Hazard-Ratios.

Im Vergleich mit der Referenzkategorie, Mütter mit niedrigem Bildungsniveau, kehren in Deutschland und in den USA die Mütter mit mittlerem und hohem Bildungsniveau insgesamt deutlich schneller in den Beruf zurück. Bei Müttern mit Abitur $(b=0,52)$ bzw. College-Erfahrung $(b=0,16)$ ist die Berufsbindung besonders hoch (Modell 1). Lediglich für Schweden finden wir hier keinen Hinweis auf einen Bildungseffekt. Insofern bestätigt sich hier nochmals das Bild aus Abb.1. Ein Vergleich der Bildungskoeffizienten in Modell 1 und 2 zeigt, dass die Bildungseffekte im zweiten Modell, in dem auf die Kontrolle des Berufsprestiges verzichtet wird, jeweils leicht stärker sind als im ersten Modell. Der Modellvergleich zeigt jedoch, dass die Unterbrechungsdauer nur in geringem Maße über die berufliche Position vermittelt wird. Das bedeutet, dass dem Bildungsniveau in Deutschland und in den USA, bei der Frage, ob und wie lange die Erwerbstätigkeit unterbrochen wird, eine zentrale Bedeutung zukommt. Da die weiteren Kontrollvariablen in allen drei Ländern für beide Modellspezifikationen stabil sind, präsentieren wir die Ergebnisse im Folgenden für diese Modelle gemeinsam. Im Unterschied zu den Bildungseffekten erklärt die Arbeitsmarkterfahrung zum Zeitpunkt der Geburt sehr wenig von der Varianz bei der Unterbrechungsdauer und erreicht nur für die USA statistisch signifikante Werte. Vorherige Erwerbsunterbrechungen in der Berufskarriere der Mütter haben in Deutschland einen positiven Effekt auf die Rückkehrrate; in Schweden und in den USA finden wir hier einen negativen Effekt. Auch das Vorhandensein weiterer Kinder im Haushalt führt in Abhängigkeit des Länderkontextes zu unterschiedlichen Ergebnissen. In Deutschland und den USA verringert sich die Rückkehrneigung für Mütter, die bereits zwei oder mehr Kinder im Haushalt haben, gegenüber Müttern mit nur einem Kind signifikant. In Schweden finden wir das entgegen gesetzte Muster: Mütter mit mehr als einem Kind im Haushalt kehren schneller ins Erwerbsleben zurück. Frauen mit Partner kehren in Schweden langsamer in den Beruf zurück als Frauen ohne Partner. Für Deutschland erhalten wir hier einen nicht signifikanten negativen Koeffizienten; weitere Analysen haben gezeigt, dass die Anzahl der Frauen, die kurz nach der Geburt des Kindes ohne Partner sind, sehr gering ist (vgl. Tab. 4 im Anhang). Das Alter bei der Geburt eines Kindes hat bei Müttern im deutschen und us-amerikanischen Sample einen negativen, bei Müttern im schwedischen Sample einen positiven Wert. Die Dummy-Variablen messen die Effekte der historisch unterschiedlich gesetzlich geförderten Unterbrechungszeiten nach der Geburt eines Kindes. Hierbei ist wegen der Proportionalitätsannahme im Cox-Modell zu beachten, dass die Größe der Koeffizienten wenig Aussagekraft besitzt, da diese die in Abb. 2 dargestellten Veränderungen über die Zeit lediglich summarisch erfasst.

Schließlich gehen wir in Modell 3 unserer dritten Hypothese entsprechend der Frage nach, ob die Ausweitung der Leave-Regelungen in den 1980er und 1990er Jahren mit regime-spezifischen Bildungseffekten bei der Inanspruchnahme von längeren Auszeiten einherging. Wir erwarten hier in Schweden und Deutschland, aufgrund des breiten Zugangs zu Elternzeit und Erziehungsgeld (bzw. Ehegattensplitting in Deutschland), eine weitgehend bildungsunabhängige Inanspruchnahme längerer Auszeiten zu finden. Für die USA erwarten wir stattdessen, Veränderungen in der Unterbrechungsdauer vor allem für Mütter mit höherem Bildungsniveau zu finden. Zur Modellierung unserer Hypothese zeigt 
Modell 3 neben den Haupteffekten für die Bildungs- und Periodendummies die entsprechenden Interaktionseffekte. Die Ergebnisse stimmen mit unseren Erwartungen überein. Für Deutschland und Schweden erreicht keiner der Interaktionseffekte das kritische Signifikanzniveau von $95 \%$. Wir finden also keinen Hinweis darauf, dass die Beanspruchung der erweiterten Elternzeitregelungen in späteren Phasen abhängig vom Bildungsniveau ist. Für die USA finden wir demgegenüber das erwartete Muster: Mütter mit Highschool Diplom und mit College-Erfahrung unterbrechen nach Einführung des FMLA häufiger ihre Erwerbstätigkeit als vor Einführung des FMLA.

Zuletzt widmen wir uns der Frage, ob die Ausweitung der Elternzeitregelungen mit bildungsschichtspezifischen Karrierekonsequenzen für Mütter in den drei Ländern assoziiert ist. Wir zeigen zunächst jeweils drei länderspezifische Modelle, in denen wir die Bildungseffekte für die Rückkehr auf gleichem Prestigeniveau diskutieren, und danach berufliche Auf- und Abstiege betrachten (Tab. 2). ${ }^{7}$ Abschließend berechnen wir noch ein Interaktionsmodell, in dem wir die Regime-Klassen-Hypothese testen (Tab.3). Wir beginnen mit der Darstellung der Ergebnisse aus Tab. 2.

Rückkehr auf gleichem Prestigeniveau. In Deutschland sowie in den USA scheint die berufliche Kontinuität abhängig vom Bildungsniveau der Mütter zu sein. Der oberste Koeffizient in der zweiten Spalte zeigt, dass Mütter mit Realschulabschluss gegenüber denen mit maximal Hauptschulabschluss eine etwa $20 \%(\operatorname{Exp}(0,19))$ höhere Chance auf berufliche Stabilität haben. Bei Müttern mit Abitur liegt der Anteil mit etwa $40 \%$ $(\operatorname{Exp}(0,34))$ doppelt so hoch. Beide Effekte sind statistisch signifikant. Dagegen finden wir für Schweden, wie erwartet, keine Bildungseffekte beim Wiedereinstieg auf gleichem Prestigeniveau. Sowohl in Deutschland als auch in den USA sind es vor allem Frauen mit höherem Schulbildungsniveau, die zum gleichen oder in einen gleichwertigen Job zurückkehren. Aufgrund der ausgedehnten Elternzeitregelungen mit Jobgarantie in Schweden und Deutschland erwarteten wir, dass die berufliche Kontinuität in beiden Ländern unabhängig vom Bildungsniveau der Mütter gewährleistet bleibt (Hypothese 3). Doch diese Erwartung bestätigt sich nur für Schweden, nicht für Deutschland. Wir gehen davon aus, dass der Bildungseffekt zumindest teilweise den extrem langen Unterbrechungsdauern in Deutschland geschuldet ist, die, wie wir in Abb. 1 gezeigt haben, in der Praxis weit über den gesetzlich geschützten Zeitraum hinausgehen. Mögliche Interpretationen dieses Befundes für Deutschland im Unterschied zu Schweden liefern wir im Abschnitt. „Diskussion der Ergebnisse“.

Berufliche Auf- und Abstiege. Auch bezogen auf berufliche Aufstiege zeigen sich in Deutschland starke Bildungseffekte. Mütter mit Abitur haben gegenüber denen mit maximal Hauptschulabschluss eine besonders hohe Wahrscheinlichkeit, bei der Rückkehr in den Arbeitsmarkt beruflich aufwärts mobil zu sein. Ein ähnliches Bild findet sich auch in Schweden, wo Aufwärtsmobilität ebenfalls nach Bildungsabschluss stratifiziert ist. Für die USA finden wir keinen signifikanten Bildungseffekt. Hierbei ist zu beachten, dass

7 Die Analysen in Tab. 2 sind in ähnlicher Form, unter Verwendung eines anderen statistischen Modells, in einem amerikanischen Journal publiziert worden (Aisenbrey et al. 2009). Wir halten es jedoch für sinnvoll, diese Ergebnisse kurz zu erläutern, da sich die in Tab. 3 präsentierten Interaktionseffekte, mit denen wir unsere vierte Hypothese abbilden, direkt auf diese Ergebnisse beziehen. 


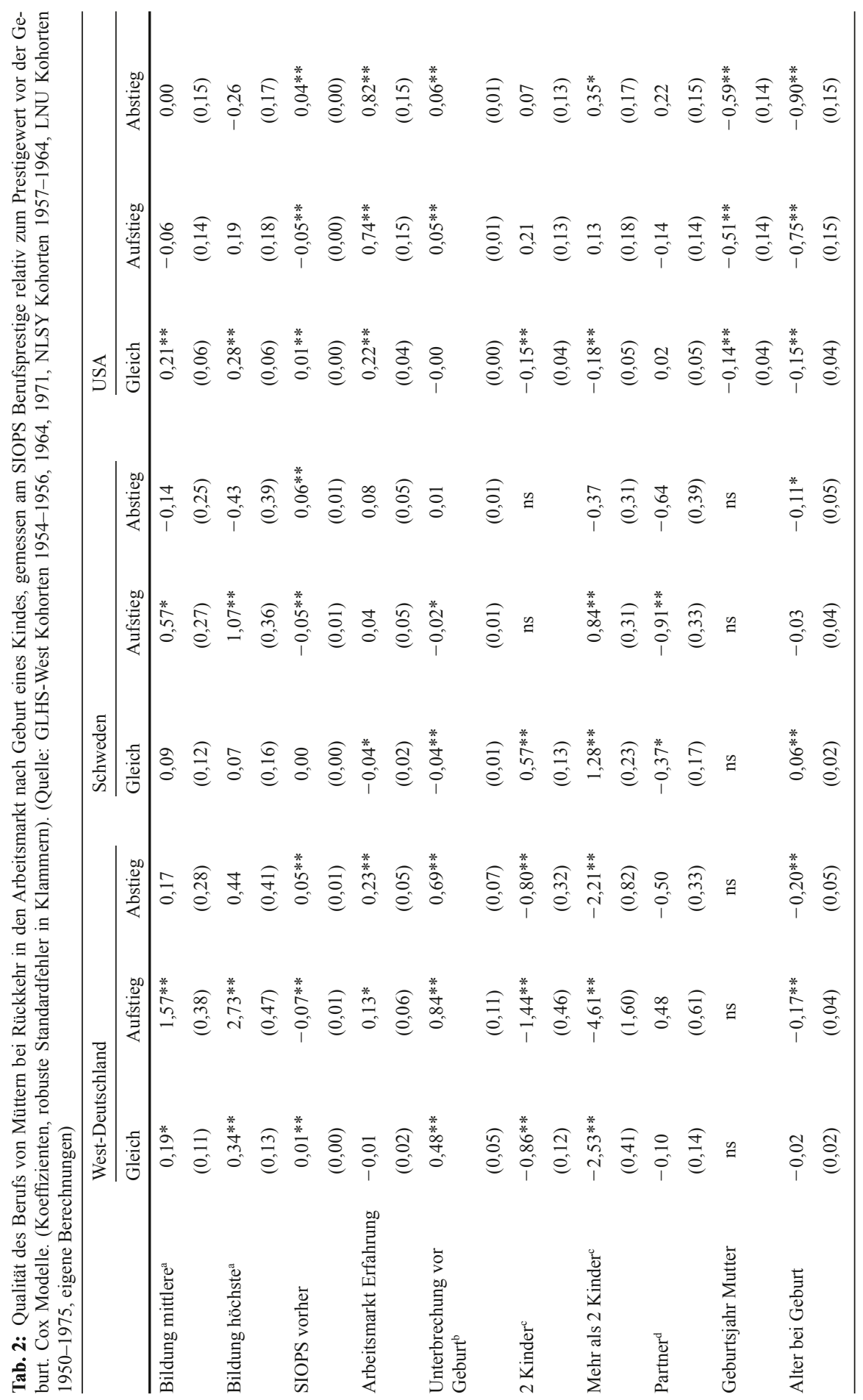




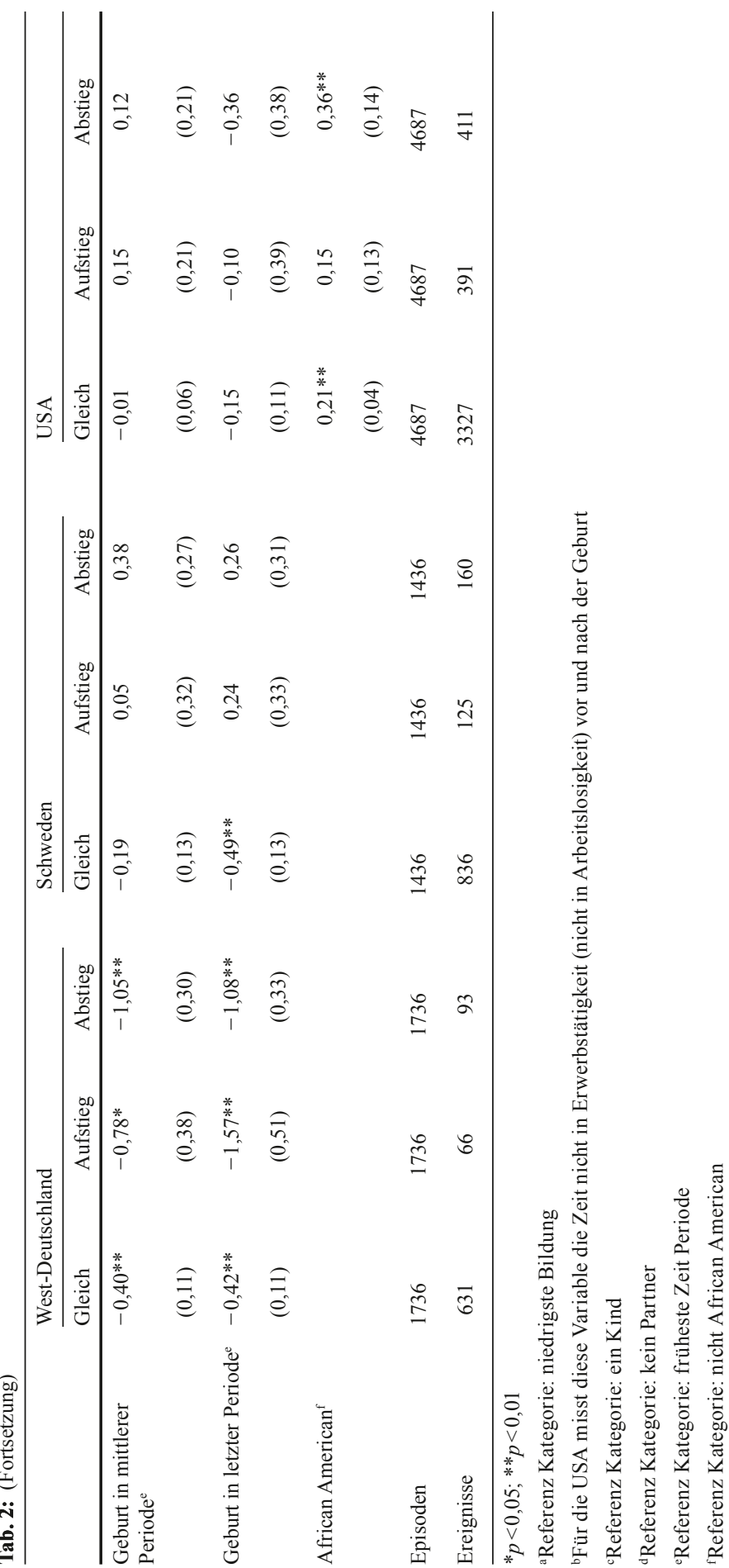




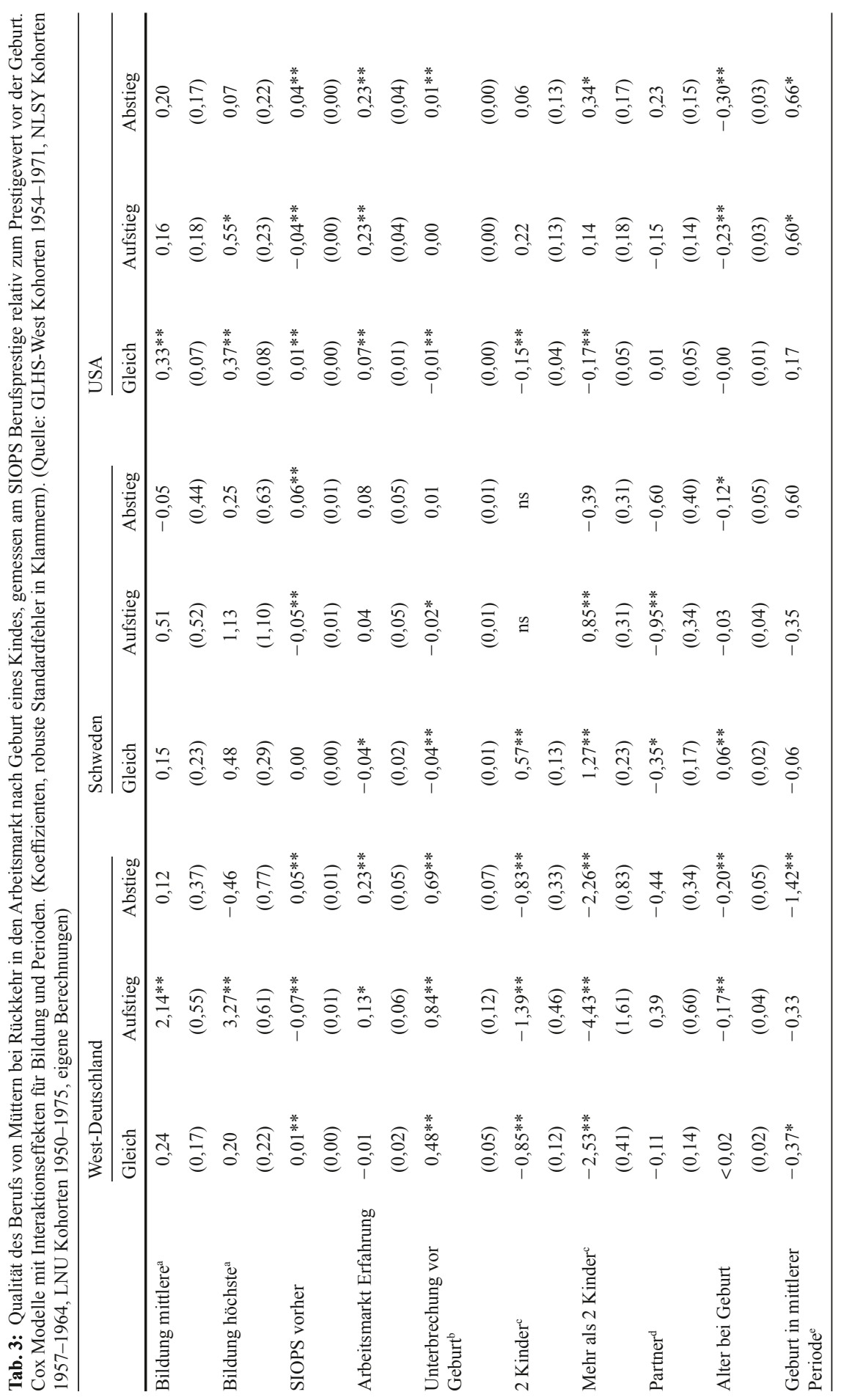




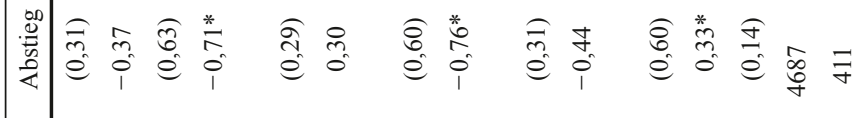

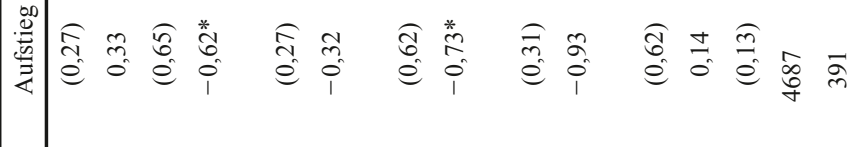

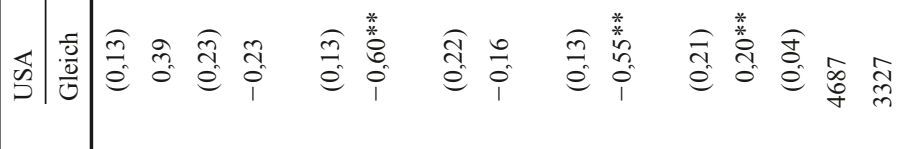

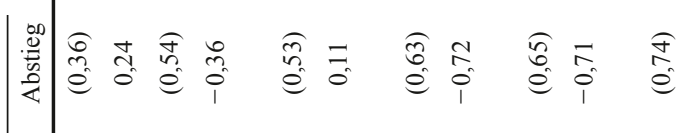

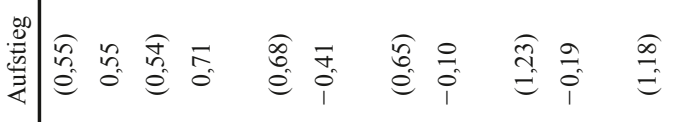

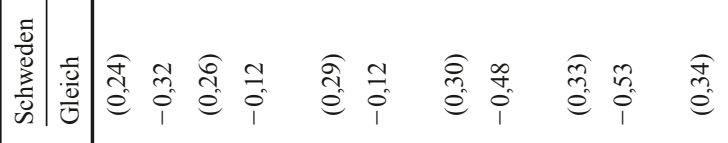

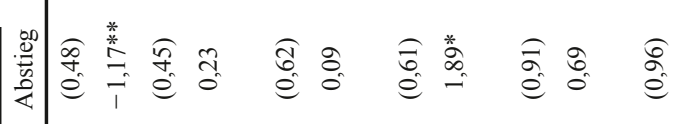

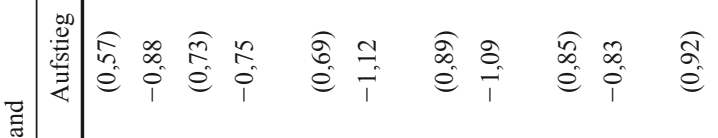

胥

咅

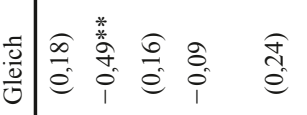

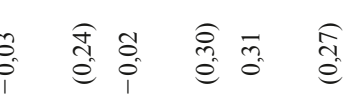

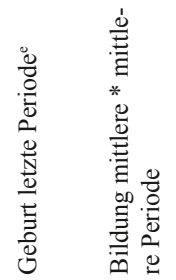

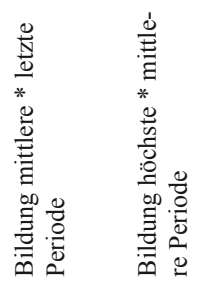

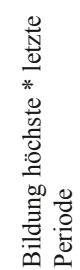

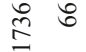

$\stackrel{\circ}{=} \Omega$

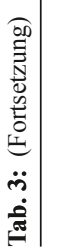

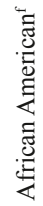

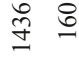

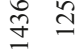

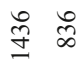

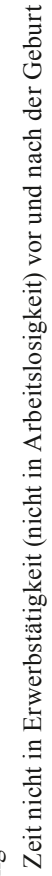

章

$\cong \overrightarrow{3}$ 
berufliche Aufstiege nach mutterschaftsbedingten Erwerbsunterbrechungen in allen drei Ländern eine Ausnahme sind. Berufliche Abstiege sind in keinem der drei Länder nach Bildungsabschlüssen stratifiziert.

Abschließend gehen wir in Anlehnung an unsere vierte Hypothese der Frage nach, ob die oben dargestellten Bildungsunterschiede bei der beruflichen Mobilität von Müttern beim Wiedereinstieg allein eine Folge längerer Unterbrechungsdauern gering qualifizierter Mütter sind, und ob die Ausweitung gesetzlicher Unterbrechungszeiten diese Unterschiede verringert oder vergrößert. Wir erwarten in Anlehnung an Mandel und Shalev (2009b), dass in Deutschland und Schweden vor allem die gering qualifizierten Mütter gegenüber den hoch qualifizierten Müttern von der Pufferwirkung des verlängerten beruflichen Statuserhalts profitieren. Die Hypothese sagt also eine Verringerung des Bildungseffekts auf die Qualität des Wiedereinstiegs über die Zeit voraus. Für die USA erwarteten wir stattdessen eine Erweiterung der Kluft zwischen den hoch und den gering Qualifizierten. Wir beschränken uns im Folgenden auf die Diskussion der Haupt- und Interaktionseffekte von Bildung und historischer Periode (Tab. 3).

Für Deutschland finden wir bestenfalls schwache Hinweise darauf, dass Mütter mit niedrigem Bildungsniveau gegenüber Müttern mit hohem Bildungsniveau von den Ausweitungen der Unterbrechungsdauern profitiert haben. Die Haupteffekte für Perioden zeigen, dass sich die Wahrscheinlichkeit beruflicher Abstiege für gering qualifizierte Mütter nach 1987 signifikant verringert hat. Die Interaktionseffekte deuten an, dass Mütter mit Abitur zwischen 1987 und 1991 zeitweilig eine signifikant höhere Neigung haben, auf niedrigerem Prestigeniveau ins Erwerbsleben zurück zu kehren, als vorher. Von den verringerten Abstiegsrisiken beim Wiedereinstieg haben zunächst also nur die geringund mittel Qualifizierten profitiert, nicht die Hochqualifizierten. In der letzten Periode (nach 1991) heben sich diese Unterschiede jedoch auf und sind nicht länger statistisch signifikant.

Die Haupteffekte für Bildung in den USA verdeutlichen, dass die Chance auf einen beruflichen Auf- oder Wiedereinstieg mit gleichem Prestige für Frauen mit höherer Bildung vor 1986 signifikant höher war als für Frauen mit niedrigem Bildungsniveau. In der darauf folgenden Periode, zwischen 1987 und 1991, kehrt sich dieser Vorsprung bei den Aufstiegschancen um. Das deuten die Interaktionseffekte für die mittlere Periode an. Dafür ist in dieser Zeit auch das Abstiegsrisiko geringer. Der einzige signifikante Bildungs-Interaktionseffekt der in der letzten Periode (nach Einführung des FMLA) für die USA ersichtlich wird, zeigt, dass Mütter mit höherer und mittlerer Bildung langsamer in Berufe auf gleichem Prestigelevel zurückkehren als in den Perioden davor. Die Neigung der hoch und mittel qualifizierten Mütter, trotz der Geburt eines Kindes kontinuierlich erwerbstätig zu sein, nimmt nach der Einführung des FMLA also wie erwartet ab. Hinweise auf eine relative Verbesserung oder Verschlechterung der Berufschancen dieser Bildungsschichten, etwa im Sinne erhöhter Auf- oder Abstiegsmobilität nach 1991, finden wir nicht. Die Ergebnisse für die USA entsprechen unserer Hypothese insofern, als die Inanspruchnahme gesetzlicher Erwerbsunterbrechungszeiten offenbar selektiv den Müttern mit Collegebildung zugute kommt, ohne für diese Gruppe mit verschlechterten Wiedereinstiegspositionen einherzugehen. Hinweise auf eine relative Verbesserung der beruflichen Wiedereinstiegsposition für hoch Qualifizierte in den USA über die Zeit finden wir jedoch nicht. 
Im Gegensatz zu Deutschland und den USA zeigen sich in Schweden keinerlei signifikante Bildungs- bzw. Periodeninteraktionseffekte. Insgesamt unterstützen unsere Ergebnisse die Regime-Klassen-Hypothese kaum. Der wesentliche Hinweis für diesen Zusammenhang findet sich in den USA, dort scheint die Inanspruchnahme von Elternzeit vielen Highschool-Abbrecherinnen nicht möglich zu sein.

\section{Diskussion der Ergebnisse}

Dieser Beitrag widmet sich der beruflichen Kontinuität von Müttern mit unterschiedlichem Schulbildungsniveau in international vergleichender Perspektive. Wir untersuchen die Strategien zur Vereinbarkeit von Berufstätigkeit und Elternschaft in (West-)Deutschland, USA und Schweden für Frauen mit unterschiedlichem Schulbildungsniveau. Wie wirkt sich länderspezifische Familienpolitik auf die Beziehung von hoch und gering qualifizierten Müttern zum Arbeitsmarkt aus? Fördern Jobgarantien die berufliche Kontinuität von Müttern unabhängig von deren Bildungsniveau oder verstärken die erweiterten Optionen der Erwerbsunterbrechung die ohnehin bestehenden sozialen Ungleichheiten zwischen den gut ausgebildeten und den gering qualifizierten Müttern? Wie sind die unterschiedlichen Familienpolitiken vor dem Hintergrund der Erwerbsmuster, die sie produzieren, im Hinblick auf die (De-)familialisierung und Kommodifizierung von Müttern zu bewerten?

Unsere Analysen beantworten diese Fragen international vergleichend auf Basis der Entwicklungen in den 1980er und 1990er Jahren. Für Deutschland ist bei der Ergebnisinterpretation zu beachten, dass die Karriereeffekte von Familienpolitik für Frauen der Geburtskohorten 1954-1956, 1964 und 1971 bis zu deren 35. Lebensjahr untersucht wurden und dass die jüngsten Entwicklungen in der deutschen Familienpolitik nach 2005 nicht einbezogen wurden. Generell wird es sicherlich noch einige Jahre dauern bis Längsschnittdaten vorliegen, die einen genügend langen Beobachtungszeitraum erfassen um die Konsequenzen dieser jüngsten Reformen in den Karriereverläufen von Müttern und Vätern abzubilden.

Unsere empirischen Ergebnisse zeigen, dass das Timing der Rückkehr an den Arbeitsplatz sowie die Konsequenzen von mutterschaftsbedingten Erwerbsunterbrechungen für den beruflichen Wiedereinstieg sowohl von den geltenden institutionellen Rahmenbedingungen als auch vom Bildungsniveau der Mütter beeinflusst werden. Die Dauer beruflicher Auszeiten von Müttern ist deutlich nach dem Bildungsniveau stratifiziert. In den USA ist annähernd jede zweite Mutter mit College Ausbildung kontinuierlich erwerbstätig; das sind fast doppelt so viele wie bei den High School Abbrecherinnen. In Schweden unterbrechen alle Mütter, unabhängig vom Bildungsniveau, für mindestens ein halbes Jahr. Danach kehren auch in Schweden die hoch ausgebildeten Mütter schneller in den Beruf zurück als die niedrig ausgebildeten. In Deutschland verzichtet jede vierte Mutter mit (Fach-)Abitur auf Elternzeit. Unsere Annahme, dass Mütter mit höherer Schulbildung, Abitur in Deutschland, College Erfahrung in den USA und Högskola in Schweden, beruflich mehr zu verlieren, aber teilweise auch mehr zu gewinnen haben, wird durch die empirischen Befunde also bestätigt. 
Auch die berufliche Kontinuität nach der Geburt eines Kindes hängt in allen drei Ländern mit dem Bildungsniveau zusammen. In den USA ist die berufliche Kontinuität, Wiedereinstieg auf gleichem Prestigeniveau, bei den Frauen mit College Erfahrung am höchsten und bei High-School Abbrecherinnen am geringsten. Gleichzeitig deuten unsere Ergebnisse für die USA darauf hin, dass es vor allem die Mütter mit Highschool und College-Erfahrung sind, die von dem im FMLA formulierten Anspruch auf eine unbezahlte Auszeit nach der Geburt eines Kindes Gebrauch machen und deshalb nach 1991 später in ihren Beruf zurückkehren. Ganz anders stellt sich die Situation in Schweden dar, wo praktisch alle Mütter, ungeachtet ihres Bildungsniveaus, ihre Erwerbstätigkeit unterbrechen. Erst nach Ablauf der gesetzlich geschützten Elternzeit zeigen sich zunehmend bildungsspezifische Rückkehrraten, nach denen die Geringqualifizierten langsamer und seltener in den Beruf zurückkehren als die hoch gebildeten Schwedinnen. Für Deutschland zeigen sich die Bildungseffekte bereits kurz nach der Geburt. Speziell im ersten Jahr nehmen Mütter mit (Fach-)Abitur deutlich kürzere Unterbrechungszeiten in Kauf als Mütter mit mittlerer Reife. Der berufliche Statuserhalt gelingt hier vor allem den gut ausgebildeten Müttern mit kurzen Unterbrechungszeiten. Wir hatten in Hypothese 3 erwartet, dass aufgrund des breiten, bildungsunabhängigen Zugangs zu Elternzeitregelungen in Deutschland und Schweden und aufgrund der Tatsache, dass diese Regelungen berufliche Kontinuität weitgehend gesetzlich garantieren, keine Bildungseffekte in diesen Ländern entstehen. Die Befunde für Deutschland zeigen jedoch, dass Mütter mit mittlerem und hohem Bildungsniveau signifikant häufiger auf gleichwertige berufliche Positionen zurückkehren als Mütter mit maximal Hauptschulabschluss (vgl. Tab.2). Gleichzeitig haben wir gezeigt, dass es anteilig vor allem die Mütter mit Hauptschulabschluss sind, die deutlich länger unterbrechen als dies in den Elternzeitprogrammen vorgesehen ist (vgl. Abb. 1). Unsere Hypothese greift aber nur unter der Voraussetzung, dass die unterschiedlich qualifizierten Mütter gleichermaßen innerhalb der für sie geltenden gesetzlichen Fristen zurück kehren. Wir gehen deshalb davon aus, dass der Bildungseffekt für Deutschland zum einen daraus resultiert, dass Wiedereinstiege von höher qualifizierten vermehrt unter dem Schutz der Elternzeitregelungen stattfinden. Zum anderen sind innerhalb der Gruppe der Mütter, die erst dann in den Arbeitsmarkt zurückkehren, nachdem ihre ,Jobgarantie“ bereits ausgelaufen ist, die besser qualifizierten gegenüber den gering qualifizierten wahrscheinlich im Vorteil. Das könnte erstens daran liegen, dass diese Frauen in unterschiedlichen Arbeitsmarktsegmenten ihren Wiedereinstieg suchen. Zweitens könnte das Ergebnis auch daran liegen, dass die Bildung ein Proxy für die soziale Klasse der Frauen ist. Wenn besser ausgebildete Frauen in finanziell besser ausgestatteten Haushalten leben, dann können sie es sich in einem konservativen Wohlfahrtsstaat eher leisten, erst dann ins Erwerbsleben zurückzukehren wenn sie einen beruflich adäquaten Job gefunden haben, der ihrer Qualifikation entspricht, als niedrig ausgebildete Frauen, deren Erwerbseinkommen in finanziell schlechter ausgestatteten Haushalten dringend benötigt wird. Diese Interpretation passt zu den Ergebnissen von Bardasi und Gornick (2000), die zeigen, dass die vorhergesagte Wahrscheinlichkeit von Frauen in Deutschland erwerbstätig zu sein, in den höheren Haushaltseinkommensperzentilen abnimmt. In Schweden stellt sich die bildungsspezifische Dynamik bezogen auf das Rückkehrverhalten von Müttern empirisch völlig anders dar. Zum einen kehrt der größte Anteil aller Mütter innerhalb der maximal gesetzlich geschützten Auszeiten in den Arbeitsmarkt zurück. Zum anderen ist auch nach 
Ablauf der gesetzlich geschützten Elternzeiten der Anteil der qualifizierten Rückkehrerinnen höher als bei den gering qualifizierten.

Die Antwort auf die Frage, ob die Ausweitung gesetzlich geschützter Erwerbsunterbrechungszeiten die bestehenden Erwerbsunterschiede für Mütter mit geringer und hoher Schulbildung verstärkt oder verringert, ist, wie unsere Analysen andeuten, Regimeabhängig. Für Schweden finden wir diesbezüglich keinen Effekt. Für Deutschland deuten die Befunde an, dass Frauen mit Abitur zeitweilig (zwischen 1987 und 1991) weniger von verringerten beruflichen Abstiegsrisiken profitierten als Frauen mit Hauptschulabschluss. Für die USA finden wir Hinweise darauf, dass Mütter mit High School Diplom und College-Erfahrung nach der Einführung des FMLA später als vor dessen Einführung auf gleichem beruflichem Niveau zurückkehren. Wir interpretieren das als Hinweis darauf, dass diese Mütter in den 1990ern verstärkt Leave beanspruchen. Die in Anlehnung an Mandel und Shalev (2009b) formulierte Regime-Klassen-Hypothese schlägt vor, dass niedrig gebildete Frauen in Schweden und Deutschland Gewinnerinnen und hochqualifizierte Frauen Verliererinnen generöser Elternzeitregelungen sind, und für die USA wird das Gegenteil erwartet. Diese Hypothese wird von unseren Ergebnissen kaum unterstützt. ${ }^{8}$

\section{Fazit und Schlussbetrachtung}

Die hier vorgelegten empirischen Ergebnisse ergänzen die bisherige Forschungsliteratur in zwei wichtigen Punkten: Erstens, die These, dass längere bezahlte Erziehungszeiten vor allem den Präferenzen gering qualifizierter Frauen in gering entlohnten und unattraktiven Berufen entsprächen, während sich Mittel- und Oberschichtfrauen am Ideal kontinuierlicher Berufstätigkeit orientierten (Morgan und Zippel 2003; Mandel und Shalev 2009b), muss im Hinblick auf die unterschiedlichen Handlungsoptionen von Frauen aus unterschiedlichen sozialen Schichten und im Kontext der wohlfahrtsstaatlichen Rahmenbedingungen gesehen werden. Unsere Ergebnisse bestätigen zwar den Bildungseffekt in allen drei Ländern, sie verweisen aber auch auf den Einfluss, den sozialstaatliche Politikpakete auf den Zeitpunkt und das Ausmaß dieses Bildungseffektes haben. In Schweden finden sich, trotz der längeren Unterbrechungszeiten von Müttern mit niedrigem Bildungsniveau, keine Bildungseffekte beim beruflichen Statuserhalt. In Deutschland hingegen sind diese Bildungseffekte deutlich ausgeprägt und der Statuserhalt gelingt Geringqualifizierten schlechter als Mittel- und Hochqualifizierten. Auch in den USA gelingt der Statuserhalt den qualifizierten Müttern deutlich besser, doch dieser Vorteil scheint davon abzuhängen, dass diese Mütter in geringerem Umfang geburtsbedingte Auszeiten beanspruchen und gleichzeitig häufiger Anspruch auf geburtsbedingte Auszeiten haben. Effekte gesetzlicher Ausweitungen der Unterbrechungszeiten sind in Deutschland und Schweden weitgehend bildungsunabhängig.

Zweitens stellt unser Befund, dass die Elternzeitregelungen in Deutschland und Schweden trotz längerer Unterbrechungszeiten den beruflichen Statuserhalt von Müttern sichern, ein wichtiges Korrektiv zu den aktuellen Befunden aus der internationa-

8 Wir können nicht ausschließen, dass unsere Befunde zumindest teilweise den geringen Fallzahlen für Schweden und Deutschland geschuldet sind. 
len Einkommensforschung dar, die vor allem den Aspekt der geschlechtsspezifischen Einkommensdifferenzen betonen und die positiven Wirkungen von Elternzeitregelungen gänzlich in Zweifel zu ziehen scheinen (Mandel und Semyonov 2005). Während es ganz unbestritten auch in Elternzeit-Ländern zu Einkommenseinbußen für Frauen und Mütter kommt, sind diese zumindest teilweise durch Teilzeitarbeit zu erklären (Bardasi und Gornick 2008) und müssen deshalb im Kontext des Haushaltseinkommens betrachtet werden. Einkommensstudien lassen jedoch nur sehr begrenzt Rückschlüsse auf die zukünftigen Chancen von Müttern zu, einen eigenständigen Haushalt zu unterhalten. Der von uns untersuchte sozioökonomische Statuserhalt sagt demgegenüber auch etwas über das Potenzial von Müttern aus, an ihre bisherige berufliche Laufbahn anzuknüpfen und so ihre Bildungsinvestitionen und berufsspezifischen Erfahrungen gegebenenfalls später im Lebenslauf wieder voll zu verwerten. Demnach unterstützen Elternzeitregelungen Mütter dabei, eine Karriere unabhängig von ihrem Partner aufzubauen und eben nicht, wie Ann Orloff es in Anlehnung an Barbara Ehrenreich (1983) bezeichnet, einen ,,husband away from poverty“" (Orloff 1993, S. 319) zu sein.

Open Access: Dieser Artikel unterliegt den Bedingungen der Creative Commons Attribution Noncommercial License. Dadurch sind die nichtkommerzielle Nutzung, Verteilung und Reproduktion erlaubt, sofern der/die Originalautor/en und die Quelle angegeben sind.

\section{Anhang: Ergänzende Angaben zu den empirischen Daten}

Tab. 4: Deskriptive Statistiken der unabhängigen Variablen, pro Episode, gemessen zu Episodenbeginn. (Quelle: GLHS-West Kohorten 1954-1956, 1964, 1971, NLSY Kohorten 1957-1964, LNU Kohorten 1950-1975, eigene Berechnungen)

\begin{tabular}{lccc}
\hline & Deutschland & USA & Schweden \\
\hline Bildung niedrigste (\%) & 41,71 & 14,08 & 20,47 \\
Bildung mittlere (\%) & 38,46 & 40,97 & 51,28 \\
Bildung höchste (\%) & 19,83 & 44,95 & 28,26 \\
Bildung total (\%) & 100 & 100 & 100 \\
Partner im HH (\%) & 92,59 & 80,94 & 96,44 \\
Geburtskohorte 1950er (\%) & 26,27 & 32,83 & 50,94 \\
Geburtskohorte 1960er (\%) & 47,18 & 67,17 & 41,14 \\
Geburtskohorte 1970er (\%) & 26,55 & & 7,92 \\
Kohorte total (\%) & 100 & 100 & 100 \\
1 Kind (\%) & 55,73 & 48,47 & 48,19 \\
2 Kinder (\%) & 34,81 & 34,67 & 36,38 \\
mehr als 2 Kinder (\%) & 9,46 & 16,86 & 15,43 \\
Kinder total (\%) & 100 & 100 & 100 \\
\hline
\end{tabular}


Tab. 4: (Fortsetzung)

\begin{tabular}{|c|c|c|c|c|c|c|}
\hline & \multicolumn{2}{|c|}{ Deutschland } & USA & \multicolumn{3}{|c|}{ Schweden } \\
\hline Geburt in frühester Periode $(\%)$ & 30,20 & & 40,75 & & 16,98 & \\
\hline Geburt in mittlerer Periode (\%) & 28,89 & & 41,13 & & 32,89 & \\
\hline Geburt in letzter Periode (\%) & 40,91 & & 18,12 & & 50,13 & \\
\hline Geburtsperiode total (\%) & 100 & & 100 & & 100 & \\
\hline $\begin{array}{l}\text { Mittelwerte (Standardabwei- } \\
\text { chungen in Klammern) }\end{array}$ & & & & & & \\
\hline SIOPS vorher & 43,19 & $(12,38)$ & 44,99 & $(14,01)$ & 39,9 & $(12,82)$ \\
\hline Arbeitsmarkt Erfahrung & 5,07 & $(3,28)$ & 7,09 & $(4,67)$ & 6,87 & $(3,84)$ \\
\hline Unterbrechung vor Geburt & 0,36 & $(1,01)$ & 2,65 & $(2,43)$ & 1,10 & $(1,59)$ \\
\hline Geburtsjahr Mutter* & & & 60,94 & $(2,04)$ & 59,7 & $(6,40)$ \\
\hline Alter bei Geburt & 27,10 & $(4,27)$ & 27,39 & $(5,10)$ & 27,87 & $(4,75)$ \\
\hline
\end{tabular}

*Für Deutschland, wegen des Kohortendesigns: Kohorte 1945-1956 26,27\%, 1964 47,18\%, 1971 $26,55 \%$

\section{Literatur}

Albrecht, James W., Per-Anders Edin, Marianne Sundström, und Susan B. Vroman. 1999. Career interruptions and subsequent earnings: a reexamination using Swedish data. Journal of Human Resources 34:294-311.

Aisenbrey, Silke, Marie Evertsson, und Daniela Grunow. 2009. Is there a career penalty for mothers' time out? Germany, Sweden, and the U.S. Compared. Social Forces 88:573-606.

Baum, Charles L. 2002. The effect of work interruptions on women's wages. Labour 16:1-37.

Bardasi, Elena, und Janet C. Gornick. 2000. Women and part-time employment: workers' , choices ' and wage penalties in five industrialized countries. Institute for Social and Economic Research (ISER): University of Essex.

Bardasi, Elena, und Janet C. Gornick. 2008. Working for less? Women's part-time wage penalties across countries. Feminist Economics 14:37-72.

Berger, Lawrence M., und Jane Waldfogel. 2004. Maternity leave and the employment of new mothers in the United States. Journal of Population Economics 17:331-349.

Boushey, Heather M. 2008. Family friendly policies: helping mothers make ends meet. Review of Social Economy 66:51-70.

Budig, Michelle J., und Paula England. 2001. The wage penalty for motherhood. American Sociological Review 66:204-225.

Bureau of Labor Statistics. 2004. U.S. Department of Labor. National longitudinal survey of young women, 1968-2003 (rounds 1-23) [computer file]. Produced and distributed by the Center for Human Resource Research. Columbus: The Ohio State University.

Datta Gupta, Nabanita, Ronald L. Oaxaca, und Nina Smith. 2003. Swimming upstream, floating downstream: comparing women's relative wage positions in the U.S. and Denmark. Bonn: IZA Discussion Papers 756.

Datta Gupta, Nabanita, und Nina Smith. 2002. Children and career interruptions: the family gap in Denmark. Economica 69:609-629. 
Dressel, Christian, Waltraud Cornelißen, und Karin Wolf. 2005. Gender-Datenreport. Kommentierter Datenreport zur Gleichstellung von Frauen und Männern in der Bundesrepublik Deutschland. München: Deutsches Jugendinstitut \& Statistisches Bundesamt.

Drobnič, Sonja. 2000. The effects of children on married and lone mothers' employment in the United States and (West) Germany. European Sociological Review 16:137-157.

Ehrenreich, Barbara. 1983. The hearts of men: American dreams and the flight from commitment. New York: Anchor Books Doubleday.

Engelbrech, Gerhard. 1997. Erziehungsurlaub - und was dann? Die Situation von Frauen bei ihrer Rückkehr auf den Arbeitsmarkt. Ein Ost/West-Vergleich. Institut für Arbeitsmarkt- und Berufsforschung Kurzbericht 8:1-5.

Engelbrech, Gerhard, und Maria Jungkunst. 2001. Erziehungsurlaub und Berufsverlauf. Informationen für die Beratungs- und Vermittlungsdienste der Bundesanstalt für Arbeit 20:1311-1322.

Esping-Andersen, Gøsta. 1990. The three worlds of welfare capitalism. Princeton: Princeton University Press.

Ferrarini, Tommy. 2006. Families, states and labour markets. Institutions, causes and consequences of family policy in post-war welfare states. Celtenham: Edward Elgar Publishing.

Gangl, Markus, und Andrea Ziefle. 2009. Motherhood, labor force behavior, and women's careers: an empirical assessment of the wage penalty for motherhood in Britain, Germany, and the United States. Demography 46:341-369.

Gornick, Janet, und Marcia Meyers. 2003. Families that work: policies for reconciling parenthood and employment. New York: Russel Sage.

Granqvist, Lena, und Helena Persson. 2004. Kvinnor och mäns karriärvägar på den svenska arbetsmarknaden. In Den könsuppdelade arbetsmarknaden, Hrsg. Åsa Löfström, SOU, 43. Stockholm: Fritzes.

Grunow, Daniela, Heather Hofmeister, und Sandra Buchholz. 2006. Late 20th-century persistence and decline of the female homemaker in Germany and the United States. International Sociology 21:101-131.

Grunow, Daniela, und Dana Müller. 2011. Zwei Kontexte - eine Kultur? Der Einfluss der Sozialisation im getrennten Deutschland von Müttern in Westdeutschland. Unveröffentlichtes Konferenzpapier. Workshop des Max-Planck-Instituts für demografische Forschung, der Universität Rostock und der Universität Bremen. Rostock: MPI.

Han, Wen J., und Christopher J. Ruhm. 2009. Parental leave policies and parents' employment and leave-taking. Journal of Policy Analysis and Management 28:548-548.

Hofferth, Sandra L., und Sally C. Curtin. 2006. Parental leave statutes and maternal return to work after childbirth in the United States. Work and Occupations 33:73-105.

Jonsson, Jan O., und Colin Mills. 2001. The sooner the better? Parental leave duration and women's occupational career. In Cradle to Grave. Life-course change in modern Sweden, Hrsg. Jan O. Jonsson und Colin Mills, 97-114. Durham: Sociologypress.

Jungwirth, Carola. 1999. Soll der Erziehungsurlaub verkürzt werden? Zeitschrift für Personalforschung 2:188-200.

Kenjoh, Eiko. 2005. New mothers' employment and public policy in the UK, Germany, the Netherlands, Sweden, and Japan. Labour 19:5-49.

Klerman, Jacob A., und Arleen Leibowitz. 1994. The work-employment distinction among new mothers. The Journal of Human Resources 29:277-303

Krüger, Helga. 2003. Berufliche Bildung: Der deutsche Sonderweg und die Geschlechterfrage. Berliner Journal für Soziologie 4:497-510.

Leitner, Sigrid, Ilona Ostner und Margit Schratzenstaller. 2004. Einleitung. In Wohlfahrtsstaat und Geschlechterverhältnis im Umbruch - Was kommt nach dem Ernährermodell? Hrsg. Sigrid Leitner, Ilona Ostner und Margit Schratzenstaller, 9-27. Wiesbaden: VS Verlag für Sozialwissenschaften. 
Lewis, Jane. 2001. The decline of the male breadwinner model: implications for work and care. Social Politics 8:152-169.

Lundberg, Shelly, und Elaine Rose. 2000. Parenthood and the earnings of married men and women. Labour Economics 7:689-710.

Mandel, Hadas, und Moshe Semyonov. 2005. Family policies, wage structures, and gender gaps: sources of earnings inequality in 20 countries. American Sociological Review 70:949-967.

Mandel, Hadas, und Moshe Semyonov. 2006. A welfare state paradox: state interventions and women's employment opportunities in 22 countries. American Journal of Sociology 111:1910-1949.

Mandel, Hadas, und Michael Shalev. 2009a. How welfare states shape the gender pay gap. Social Forces 87:1873-1911.

Mandel, Hadas, und Michael Shalev. 2009b. Gender, class, and varieties of capitalism. Social Politics 16:161-181.

Mayer, Karl U. 2008. Retrospective longitudinal research: the German life history study. In Handbook of longitudinal research: design, measurement and analysis, Hrsg. Scott Menard, 85106. San Diego: Elsevier.

Mincer, Jacob, und Solomon Polachek. 1974. Family investments in human capital: earnings of women. The Journal of Political Economy 82:S76-S108.

Misra, Joya, Michelle J. Budig, und Stephanie Moller. 2007. Reconciliation policies and the effects of motherhood on employment, earnings and poverty. Journal of Comparative Policy Analysis 9:135-155.

Morgan, Kimberly J., und Kathrin Zippel. 2003. Paid to care: the origins and effects of care leave policies in western Europe. Social Politics 10:49-85.

Moss, Peter, und Karin Wall. 2007. International review of leave policies and related research 2007. Employment Relations Research Series 80.

O'Reilly, Jaqueline, und Colette Fagan. 1998. Part-time prospects: an international comparison of part-time work in Europe, North America, and the Pacific Rim. London: Routledge.

Orloff, Ann S. 1993. Gender and the social rights of citizenship: the comparative analysis of gender relations and welfare states. American Sociological Review 58:303-328.

Orloff, Ann S. 2009. Should feminists aim for gender symmetry? Why the dual-earner/dual-carer model may not be every feminist's utopia. In Gender equality, transforming family divisions of labor, Hrsg. Janet Gornick und Marcia Meyers, 129-160. New York: Verso.

Ostner, Ilona, und Jane Lewis. 1998. Geschlechterpolitik zwischen europäischer und nationalstaatlicher Regelung. In Standort Europa. Sozialpolitik zwischen Nationalstaat und europäischer Integration, Hrsg. Stephan Leibfried und Paul Pierson, 196-239. Frankfurt a. M.: Suhrkamp.

Pettit, Becky, und Jennifer Hook. 2005. The structure of women's employment in comparative perspective. Social Forces 84:779-801.

Ray, Rebecca, Janet C. Gornick, und John Schmitt. 2009. Parental leave policies in 21 countries: assessing generosity and gender equality, center for economic and policy research. Washington, DC: Center for Economic and Policy Research.

Ruhm, Christopher J. 1998. The economic consequences of parental leave mandates: lessons from Europe. Quarterly Journal of Economics 113:285-317.

Treiman, Donald J. 1977. Occupational prestige in comparative perspective. New York: Academic Press.

United Nations Statistics Division. 2010. Statistics and indicators on women and men: part-time employment. New York. Web: http://unstats.un.org/unsd/demographic/products/indwm/tab5b. htm. Zugegriffen: 15. Apr. 2011.

Waldfogel, Jane. 2001. International policies toward parental leave and child care. The Future of Children 11:98-111.

Yoest, Charmaine. 2003. The family, gender and tenure project, University of Virginia, Projekt Bericht der Alfred Sloan Foundation. http://faculty.virginia.edu/familyandtenure/institutional\%20report.pdf. Zugegriffen: 15. Apr. 2011. 
Ziefle, Andrea. 2004. Die individuellen Kosten des Erziehungsurlaubs: Eine empirische Analyse der kurz- und längerfristigen Folgen für den Karriereverlauf von Frauen. Kölner Zeitschrift für Soziologie und Sozialpsychologie 56:213-231.

Daniela Grunow, 1975, Dr. rer. pol., Associate Professor of Sociology an der Universiteit van Amsterdam, NL und Associate Fellow am Center for Research on Inequalities and the Life Course, Yale University, USA. Forschungsgebiete: Sozialstruktur und soziale Ungleichheit im internationalen Vergleich, Geschlecht, Familie und Lebenslauf, Methoden der empirischen Sozialforschung. Veröffentlichungen: Comparing Diary and Survey Estimates on Time Use. European Sociological Review. Online-first doi:10.1093/esr/jcr030, 2011 (mit F. Schulz). Mythos Flexibilisierung? Wie instabil sind Arbeitsbiografien wirklich und als wie instabil werden sie wahrgenommen? Kölner Zeitschrift für Soziologie und Sozialpsychologie. 62, 2010 (mit K. U. Mayer und N. Nitsche). Is there a Career Penalty for Mothers' Time Out? Germany, Sweden and the U.S. compared. Social Forces 88, 2009 (mit S. Aisenbrey und M. Evertsson). Was erklärt die Traditionalisierungsprozesse häuslicher Arbeitsteilung im Eheverlauf: soziale Normen oder ökonomische Ressourcen? Zeitschrift für Soziologie 36, 2007 (mit F. Schulz und H.-P. Blossfeld)

Silke Aisenbrey, 1971, Dr., Assistant Professor of Sociology an der Yeshiva University, New York, USA. Forschungsgebiete: Soziale Ungleichheit, Lebensverlaufsforschung, Vergleichende Wohlfahrtsstaatsforschung und Methoden der empirischen Sozialforschung. Veröffentlichungen: New life for old ideas: The ,Second Wave' of Sequence Analysis Bringing the ,Course' Back into the Life Course. Sociological Methods and Research 38/3 2010 (mit A. Fasang). Is there a Career Penalty for Mothers' Time Out? Germany, Sweden and the U.S. compared. Social Forces 88, 2009 (mit S. Aisenbrey und M. Evertsson). Penalties and Rewards of Family Formation: Poverty Risk and Status Passages in Family, Work and Education in Germany. British Journal of Sociology 60/1, 2009

Marie Evertsson, 1969, PhD, Associate Professor (Docent) of Sociology am Swedish Institute for Social Research, Stockholm University, SWE, Mitgleid des Stockholm University Linnaeus Center on Social Policy and Family Dynamics in Europe und Associate Fellow am Center for Research on Inequalities and the Life Course, Yale University, USA. Forschungsgebiete: Soziale Ungleichheit im internationalen Vergleich, Geschlecht und Familie. Veröffentlichungen: Is there a Career Penalty for Mothers' Time Out? Germany, Sweden and the U.S. compared. Social Forces 88, 2009 (mit S. Aisenbrey und D. Grunow). Parental Leave - Possibility or Trap? Does Family Leave Length Affect Swedish Women's Labor Market Opportunities? European Sociological Review. Online-first doi: 10.1093/esr/jcq018, 2010 (mit A.-Z. Duvander). Is Gender Inequality Greater at Lower or Higher Educational Levels? Common Patterns in the Netherlands, Sweden and the U.S. Social Politics 16: 210-241 (mit P. England, I. Reci, J. Hermsen, J. de Bruijn and D. Cotter) 